\title{
The Toda system and clustering interfaces in the Allen-Cahn equation
}

\author{
Manuel del Pino *Michał Kowalczyk † Juncheng Wei ${ }^{\ddagger}$
}

\begin{abstract}
We consider the Allen-Cahn equation $\varepsilon^{2} \Delta u+\left(1-u^{2}\right) u=0$ in a bounded, smooth domain $\Omega$ in $\mathbb{R}^{2}$, under zero Neumann boundary conditions, where $\varepsilon>0$ is a small parameter. Let $\Gamma_{0}$ be a segment contained in $\Omega$, connecting orthogonally the boundary. Under certain non-degeneracy and non-minimality assumptions for $\Gamma_{0}$, satisfied for instance by the short axis in an ellipse, we construct, for any given $N \geq 1$, a solution exhibiting $N$ transition layers whose mutual distances are $O(\varepsilon|\log \varepsilon|)$ and which collapse onto $\Gamma_{0}$ as $\varepsilon \rightarrow 0$. Asymptotic location of these interfaces is governed by a Toda type system and yields in the limit broken lines with an angle at a common height and at main order cutting orthogonally the boundary.
\end{abstract}

\section{Introduction}

Let $\Omega$ be a bounded domain with smooth boundary in $\mathbb{R}^{2}$. In this paper we consider the elliptic problem:

$$
\begin{gathered}
\varepsilon^{2} \Delta u+\left(1-u^{2}\right) u=0 \quad \text { in } \Omega, \\
\frac{\partial u}{\partial \nu}=0 \quad \text { on } \partial \Omega .
\end{gathered}
$$

Here $\varepsilon>0$ is a small parameter and $\nu$ denotes unit outer normal to $\partial \Omega$. Equation (1.1)-(1.2) is known as the Allen-Cahn equation and was introduced in [2] as a model describing the evolution of antiphase boundaries.

Problem (1.1)-(1.2) and its parabolic counterpart have been a subject of extensive research for many years. Its solutions correspond to critical points in $H^{1}(\Omega)$ of the Allen-Cahn energy,

$$
J_{\varepsilon}(u)=\int_{\Omega}\left[\frac{\varepsilon}{2}|\nabla u|^{2}+\frac{1}{4 \varepsilon}\left(1-u^{2}\right)^{2}\right] .
$$

We observe that the states $u= \pm 1$ represent both global minimizers of the energy, which in the Allen-Cahn model correspond to two phases of a material isolated in the region $\Omega$. We are interested in solutions which connect these states, leaving an interface, a narrow region where the

\footnotetext{
* Manuel del Pino - Departamento de Ingeniería Matemática and CMM, Universidad de Chile, Casilla 170 Correo 3, Santiago, Chile. Email: delpino@@dim.uchile.cl

†Michał Kowalczyk - Departamento de Ingeniería Matemática and CMM, Universidad de Chile, Casilla 170 Correo 3, Santiago, Chile. Email kowalczy@@dim.uchile.cl

$\ddagger$ Juncheng Wei - Department of Mathematics, Chinese University of Hong Kong, Shatin, Hong Kong Emailwei@@math.cuhk.edu.hk
} 
transition from one phase to the other takes place. More precisely, we consider solutions $u_{\varepsilon}$ to (1.1)-(1.2) for which $J\left(u_{\varepsilon}\right)$ remains uniformly bounded, which formally entails a uniform bound for the length of the transition region. These solutions approach \pm 1 almost everywhere as $\varepsilon \rightarrow 0$ while, at least for minimizers, the energy values approach perimeter of the limiting interface between the regions where values +1 and -1 are taken. In the language of $\Gamma$-convergence, the $\Gamma$-limit of $J_{\varepsilon}$ corresponds precisely to interface perimeter, see [22, 29, 38].

In [22] Kohn and Sternberg constructed local minimizers using this fact. Associated to a straight line segment $\Gamma_{0}$ contained in $\Omega$ which locally minimizes length among all curves nearby with endpoints lying on $\partial \Omega$, they find a local minimizer $u_{\varepsilon}$ of $J_{\varepsilon}$ with asymptotic interface given by this segment. One has

$$
J_{\varepsilon}\left(u_{\varepsilon}\right) \rightarrow c_{0}\left|\Gamma_{0}\right|
$$

for some universal constant $c_{0}>0$. Other qualitative properties of minimizers can be found in $[5,6,16,35,39]$.

It is natural to ask for existence and asymptotic description of solutions other than local minimizers. We recall for instance that no local minimizers other than constants are present if the domain is convex $[7,30]$. On the other hand rich phenomena should be observable even in this case, as heat flow for the Allen-Cahn equation becomes in the appropriate limit mean curvature flow of interfaces, see [3, 36, 8, 9, 15, 18, 37, 21].

In $[35,40,17]$ the authors have addressed the issue of understanding asymptotic behavior of interfaces for general families of solutions of (1.1)-(1.2) with uniformly bounded energy through a geometric measure theoretical approach. Roughly speaking, they have established that asymptotic interfaces must be locally stationary for perimeter. More generally, in higher dimensions, they correspond locally to (generalized) minimal hypersurfaces. However, not many concrete cases where such objects can be found have been known. Part of the reason is that, once minimization is not available, traditional variational tools do not yield existence or fine analysis easily.

Along these lines, it is natural to consider a situation like that of Kohn and Sternberg [22] for a critical, not necessarily minimizing segment. We assume in what follows that $\Omega$ contains a straight line segment $\Gamma_{0}$ which intersects orthogonally the boundary at exactly two points $P_{0}, P_{1} \in \partial \Omega$.

We assume that $\Gamma_{0}$ is non-degenerate in the sense that if $\kappa(P), P \in \partial \Omega$, represents curvature of the boundary, then the following condition holds:

$$
\kappa\left(P_{0}\right)+\kappa\left(P_{1}\right)-\kappa\left(P_{0}\right) \kappa\left(P_{1}\right)\left|\Gamma_{0}\right| \neq 0 .
$$

Let us explain the meaning of this condition. After translation and rotation we may assume that $\Gamma_{0}$ is given by

$$
\Gamma_{0}=\left\{\left(x_{1}, x_{2}\right) \mid x_{1}=0,0<x_{2}<\ell\right\}
$$

where $\ell=\left|\Gamma_{0}\right|$. Let us assume that near the endpoints of the segment, $\partial \Omega$ is described as the graph of two smooth functions, respectively $x_{2}=G_{0}\left(x_{1}\right), x_{2}=G_{1}\left(x_{1}\right)$, with $G_{0}(0)=$ $0, G_{1}(0)=\ell, G_{0}^{\prime}(0)=0=G_{1}^{\prime}(0)$, so that $G_{0}^{\prime \prime}(0)=\kappa\left(P_{0}\right), G_{1}^{\prime \prime}(0)=-\kappa\left(P_{1}\right)$. Any curve $C^{1}$-close to $\Gamma$ with endpoints on $\partial \Omega$ can be parametrized as

$$
\gamma(t)=\left(h(t), t G_{1}(h(\ell))+(\ell-t) G_{0}(h(0))\right), \quad t \in[0, \ell] .
$$

where $h:[0, \ell] \rightarrow \mathbb{R}^{2}$ represents a small function of class $C^{1}$. The length of this curve is then given by the functional on $h$,

$$
|\gamma|=\rho(h)=\int_{0}^{\ell} \sqrt{\left[G_{1}(h(\ell))-G_{0}(h(0))\right]^{2}+h^{\prime}(t)^{2}} d t
$$


so that in particular its first variation $D \rho(0)=0$. In fact, criticality of the straight segment means just that it intersects orthorgonally the boundary. Second variation of length is then given by the quadratic form

$$
D^{2} \rho(0)[h]^{2}=\int_{0}^{\ell} h^{\prime}(t)^{2} d t+G_{1}^{\prime \prime}(0) h(\ell)^{2}-G_{0}^{\prime \prime}(0) h(0)^{2} .
$$

Non-degeneracy of $\Gamma_{0}$ means precisely that this quadratic form is non-degenerate in its natural space $H^{1}(0, \ell)$. This is equivalent to the fact that the problem

$$
\begin{gathered}
-h^{\prime \prime}=\lambda h, \quad \text { in }(0, \ell), \\
\kappa\left(P_{0}\right) h(0)+h^{\prime}(0)=0, \\
-\kappa\left(P_{1}\right) h(\ell)+h^{\prime}(\ell)=0,
\end{gathered}
$$

does not have $\lambda=0$ as an eigenvalue. A direct computation shows that the latter fact is equivalent to condition (1.4). Let us observe that if both curvatures are negative the second variation is positive and we are in the minimizing situation of [22].

The segment $\Gamma_{0}$ separates $\Omega$ into two subdomains $\Omega_{-}, \Omega_{+}$, respectively to the left and to the right of it. In [24] the second author has established that if (1.4) holds then there exists a solution $u_{\varepsilon}$ to (1.1)-(1.2) exhibiting a transition layer at a distance $O(\varepsilon)$ of $\Gamma_{0}$. More precisely, if $s$ is the signed distance to $\Gamma_{0}$, then these solutions are well approximated by $U(s / \varepsilon)$ where $U$ is the unique solution of the problem

$$
\begin{aligned}
& U^{\prime \prime}+\left(1-U^{2}\right) U=0, \quad \text { in } \mathbb{R}, \\
& U( \pm \infty)= \pm 1, \quad U(0)=0 .
\end{aligned}
$$

One has for this solution $J_{\varepsilon}\left(u_{\varepsilon}\right) \rightarrow c_{0}\left|\Gamma_{0}\right|$, just as in the $\Gamma$-convergence situation of Kohn and Sternberg (1.3) and

$$
\lim _{\varepsilon \rightarrow 0} u_{\varepsilon}= \pm 1 \quad \text { in } \Omega_{ \pm} .
$$

The purpose of this paper is to show that in the non-minimizing situation for the segment $\Gamma_{0}$ much richer phenomena are actually present.

Before stating our results, let us discuss a bit further the nondegeneracy condition (1.4). Let us assume that, opposite to the minimizing situation both curvatures $\kappa\left(P_{0}\right), \kappa\left(P_{1}\right)$, are positive, just as in the case of the short axis or the long axis of an eccentric ellipse. In that case, parallel translation of the segment reduces the length of its portion inside $\Omega$. On the other hand, rotation of the short axis around its center increases length inside $\Omega$, while that of the short axis degreases it: this translates into the fact that the short axis produces a non-degenerate critical point of Morse index one for length (namely only one direction to decrease length is present), while the long axis represents one of Morse index two.

In general, as direct computation of eigenvalues of problem (1.6) shows, when both curvatures are positive, we have that exactly one eigenvalue is negative if

$$
\kappa\left(P_{0}\right)+\kappa\left(P_{1}\right)-\kappa\left(P_{0}\right) \kappa\left(P_{1}\right)\left|\Gamma_{0}\right|>0
$$

which we call the "short axis situation", while exactly two of these eigenvalues are negative if

$$
\kappa\left(P_{0}\right)+\kappa\left(P_{1}\right)-\kappa\left(P_{0}\right) \kappa\left(P_{1}\right)\left|\Gamma_{0}\right|<0,
$$

the "long axis situation". By definition the numbers

$$
R_{0}=\frac{1}{\kappa\left(P_{0}\right)}, \quad R_{1}=\frac{1}{\kappa\left(P_{1}\right)}
$$


correspond to the radii of the osculating circles to $\partial \Omega$ respectively at $P_{0}$ and $P_{1}$, so that condition (1.8) becomes just

$$
R_{1}+R_{2}>\ell .
$$

As we will see, under this condition and the additional assumption

$$
\left|R_{1}-R_{2}\right|<\ell
$$

which in particular holds for the short axis of an ellipse, there exist solutions with multiple interfaces. In fact, given $N \geq 1$, there is a solution $u_{\varepsilon}$ to problem (1.1)-(1.2) such that

$$
\begin{gathered}
J_{\varepsilon}\left(u_{\varepsilon}\right) \rightarrow N c_{0}\left|\Gamma_{0}\right| \quad \text { as } \varepsilon \rightarrow 0, \\
\lim _{\varepsilon \rightarrow 0} u_{\varepsilon}=-1 \quad \text { in } \Omega_{-}, \quad \lim _{\varepsilon \rightarrow 0} u_{\varepsilon}=\left\{\begin{array}{c}
1 \text { if } N \text { is odd } \\
-1 \text { if } N \text { is even }
\end{array} \quad \text { in } \Omega_{+} .\right.
\end{gathered}
$$

These are solutions exhibiting $N$ transition layers, close and approximately parallel to each other, which eventually collapse onto $\Gamma_{0}$.

An interesting feature of these solutions is the role played by the Toda system in the asymptotic location of their multiple interfaces. Let us recall that the Toda system describes dynamics of $N$ particles, arranged on a line and interacting with their neighbors with a force proportional to the exponential of minus their mutual distance. Considering interfaces, we observe that two nearly parallel interfaces attract, in the sense that as they get closer to one another, energy decreases proportionally to the exponential of the negative of their mutual distance. On the other hand, because of assumption (1.11), interfaces raise their individual energies proportionally to their length as they approach horizontally the segment. It is therefore expected that an equilibrium location, maximizing effect due to interactions in parallel motion and minimizing in length should exist. In particular, interfaces in such an equilibrium should prefer to stay parallel since rotation increases length in the short axis situation. This is why Toda system appears, in which the segment coordinate takes the role of time.

We should take into account that at a closer look, these multiple interfaces should not stay quite as straight lines since they need to arrive orthogonally to the boundary near the upper and lower ends. In reality, analysis of the Toda system under boundary conditions giving account of this orthogonality, shows that interfaces correspond asymptotically to broken lines of the form

$$
x_{1}=\mu\left(\left|x_{2}-\gamma_{0}\right|+\gamma_{1}\right), \quad \mu=O(\varepsilon \log \varepsilon) .
$$

These curves intersect orthogonally the respective osculating circles for $P_{0}$ and $P_{1}$ precisely if

$$
\gamma_{0}=\frac{1}{2}\left(\ell-R_{1}+R_{0}\right), \quad \gamma_{1}=\frac{1}{2}\left(R_{1}+R_{0}-\ell\right) .
$$

Observe that relations $(1.11),(1.12)$ correspond to the facts

$$
\gamma_{0} \in(0, \ell), \quad \gamma_{1}>0 .
$$

$\gamma_{0}$ is the $x_{2}$-coordinate of the midpoint of the segment between the centers of the two osculating circles, which we thus assume lies on $\Gamma_{0}$. Assuming $\gamma_{1}>0$ implies that the broken lines (1.15) do not touch $\Gamma_{0}$.

Our main result reads as follows.

Theorem 1.1 Assume that the segment $\Gamma_{0}$ given by (1.5) is such that $\kappa\left(P_{j}\right)>0, j=0,1$ and that conditions (1.11) and (1.12) hold. Then for each $N>1$ and all sufficiently small $\varepsilon$, there exists a solution $u_{\varepsilon}$ to problem (1.1)-(1.2) which satisfies relations (1.13), (1.14), and in a neighborhood of $\Gamma_{0}$ it has the form

$$
u_{\varepsilon}\left(x_{1}, x_{2}\right)=o(1)+\sum_{k=1}^{N}(-1)^{k+1} U\left(\frac{x_{1}-\varepsilon f_{k}\left(x_{2}\right)}{\varepsilon}\right)+\left\{\begin{array}{r}
0 \text { if } N \text { is odd } \\
-1 \text { if } N \text { is even }
\end{array}\right.
$$


with the functions $f_{k}, k=1, \ldots, N$ satisfying the asymptotic expression

$$
f_{k}\left(x_{2}\right)=c_{0}|\log \varepsilon|\left(k-\frac{N+1}{2}\right)\left(\left|x_{2}-\gamma_{0}\right|+\gamma_{1}+o(1)\right) .
$$

Here $\gamma_{0}, \gamma_{1}$ are the constants given by formula (1.16), $c_{0}>0$ is a universal constant and the quantities o(1) go to zero uniformly as $\varepsilon \rightarrow 0$.

We include some pictures for different values of the parameters of the approximate shape of the interfaces in Figures 1 and 1 below.

We can be much more precise in the way interfaces satisfying expansion (1.18) arise. It turns out that functions $f_{k}$ solve at main order the Toda system

$$
\varepsilon^{2} f_{k}^{\prime \prime}=a_{0}\left[e^{-\sqrt{2}\left(f_{k}-f_{k-1}\right)}-e^{-\sqrt{2}\left(f_{k+1}-f_{k}\right)}\right] \quad \text { in }(0, \ell)
$$

under boundary conditions

$$
f_{k}^{\prime}(0)+\kappa\left(P_{0}\right) f_{k}(0)=0=f_{k}^{\prime}(\ell)-\kappa\left(P_{1}\right) f_{k}(\ell), \quad k=1, \ldots, N,
$$

for some universal constant $a_{0}>0$ and with the conventions $f_{0}=-\infty, f_{N+1}=+\infty$. As we will later justify, this problem has a unique solution, whose expansion as $\varepsilon \rightarrow 0$ corresponds to (1.18).

The presence of higher multiplicity interfaces has been first suggested in [3]. In the one dimensional or radial cases, concentration in the form of clusters of transition layers or spikes has already been observed in various problems in the literature, see [4, 10, 11, 13, 32, 33, 27, 28], phenomena in accordance with higher-dimensional multi-spike clustering as predicted in [20]. In particular, multiple radial transition spheres collapsing on the boundary have been found for Allen-Cahn in [28]. The result of Theorem 1.1 seems to be the first of its type in a fully higher dimensional setting. Single-higher dimensional concentration phenomena has been the subject in, among other works, $[25,26,12]$.

A broad literature exists for the Toda system, including various representation formulas for their solutions, we refer the reader to $[19,23,31]$ for results and references. The link found here seems new, and we expect that classical mechanical systems are in agreement with various multiple-curve concentration phenomena arising in elliptic singular perturbations, in similar way as gravitational or electrostatic interpretation of point concentration is commonly given.

In [34], Allen-Cahn in dimensions 3 or higher in a compact manifold has been considered, establishing that associated to a non-degenerate minimal hypersurface a solution with a single interface exists, in analogy with the result in [24]. We would expect multiple concentration of codimension one interfaces dimensions two and higher to exist, but with more difficult proofs.

The proof of Theorem 1.1 consists of finding a solution close to an initial approximation, which is essentially the right hand side of expression (1.17). The correction term satisfies an equation which is solved in two steps: first the curves $f_{k}$ are just left as parameters to be determined, and a projected problem is solved which involves a small nonlinear perturbation of a uniformly invertible operator in suitable norms. Second, functions $f_{k}$ are chosen in such a way that the solution of the projected problem is a full solution. In this second part of the process a small nonlinear, nonlocal perturbation of system (1.19)-(1.20) arises, which is solved thanks to nondegeneracy of the solution of the unperturbed system. We shall develop this scheme in what remains of this paper, in which hypotheses of Theorem 1.1 will always be assumed. 

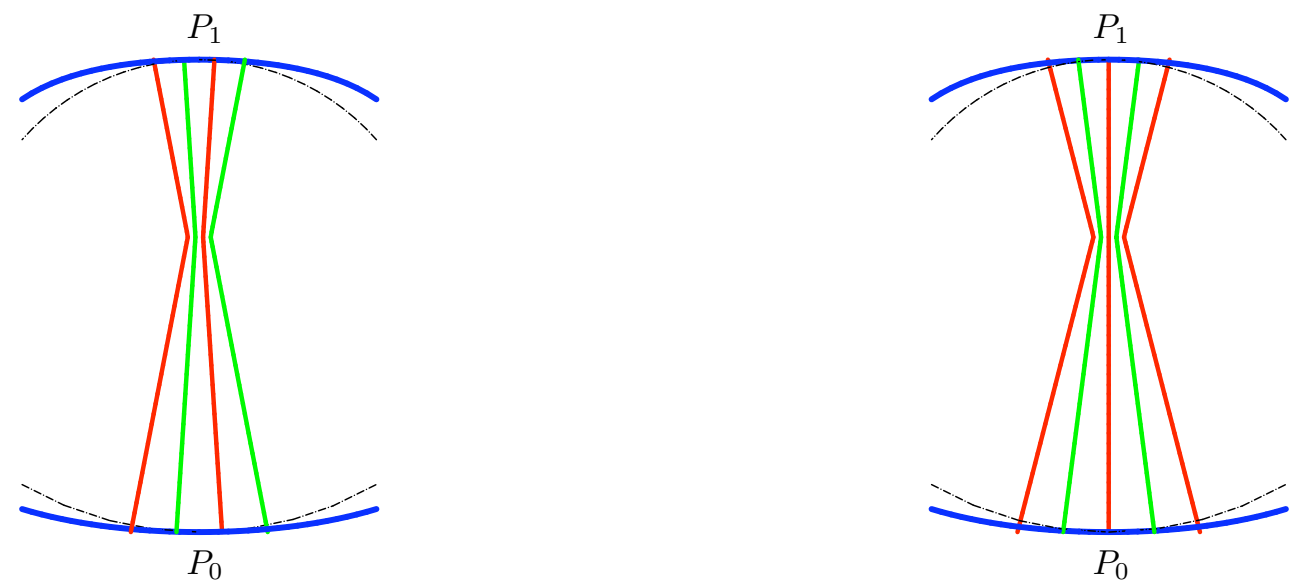

Figure 1: Equilibrium configurations of 4 and 5 interfaces with $\ell=1, R_{0}=\frac{3}{4}, R_{1}=\frac{1}{2}$ and $\varepsilon=\frac{1}{25}$. The dotted lines indicate the osculating circles of the boundary at points $P_{0}$ and $P_{1}$ respectively.
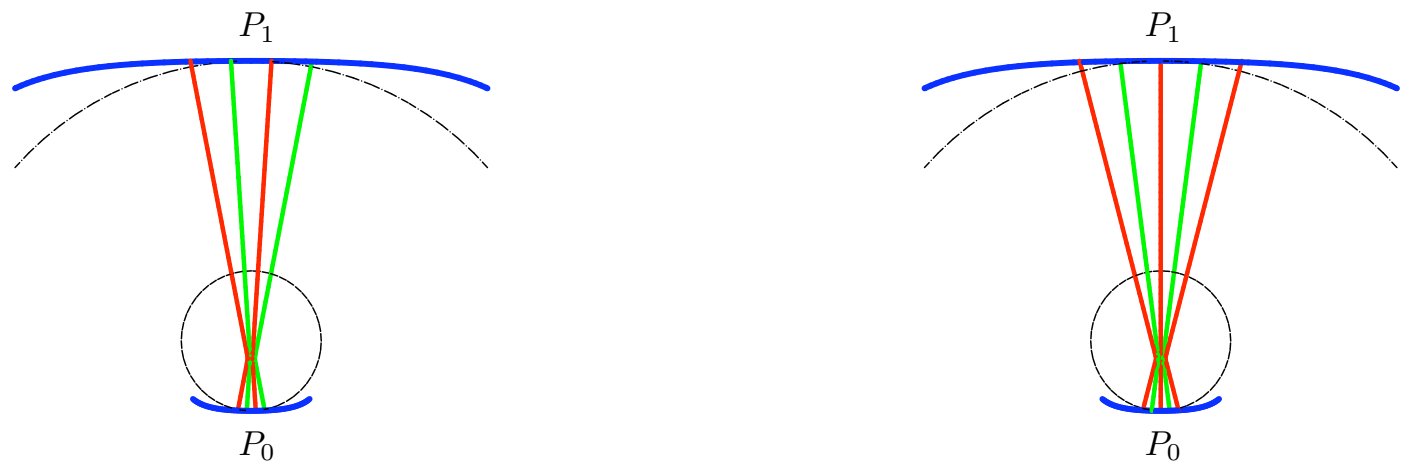

Figure 2: Equilibrium configurations as in Figure 1 with $\ell=1, R_{0}=\frac{1}{5}, R_{1}=\frac{9}{10}$ and $\varepsilon=\frac{1}{25}$. 


\section{First approximation and outline of the proof}

\subsection{Approximate solution}

We first formulate our problem in conveniently chosen system of coordinates. With no loss of generality, we may assume that the segment $\Gamma_{0}$ satisfies $\left|\Gamma_{0}\right|=1$ and is given by

$$
\Gamma_{0}=\left\{\left(x_{1}, x_{2}\right) \mid x_{1}=0,0<x_{2}<1\right\} .
$$

We also assume that near the endpoints of the segment, $\partial \Omega$ is described as the graph of two smooth functions, let us say respectively $x_{2}=G_{0}\left(x_{1}\right), x_{2}=G_{1}\left(x_{1}\right)$, with

$$
G_{0}(0)=0, \quad G_{1}(0)=1, \quad G_{0}^{\prime}(0)=0=G_{1}^{\prime}(0) .
$$

Let us consider the scaling $v(y)=u(\varepsilon y)$. Problem (1.1)-(1.2) is thus equivalent to

$$
\begin{array}{rll}
\alpha(v) \equiv \Delta v+f(v)=0 & \text { in } \Omega_{\varepsilon} \\
\beta(v) \equiv \frac{\partial v}{\partial \nu}=0 & \text { on } \partial \Omega_{\varepsilon} .
\end{array}
$$

where $\Omega_{\varepsilon}=\varepsilon^{-1} \Omega$. Here and in what follows we denote

$$
f(v)=\left(1-v^{2}\right) v
$$

For some small, fixed number $\delta_{0}$, we can describe diffeomorphically all points $y \in \Omega_{\varepsilon}$ with $\left|y_{1}\right|<\delta_{0} \varepsilon^{-1}$ by means of coordinates $(x, z)$ which straighten the boundary as follows.

$$
x=y_{1}, \quad z=y_{2}-\eta_{\sigma}\left(\varepsilon y_{2}\right) \varepsilon^{-1} G_{0}\left(\varepsilon y_{1}\right)-\eta_{\sigma}\left(1-\varepsilon y_{2}\right) \varepsilon^{-1}\left[G_{1}\left(\varepsilon y_{1}\right)-1\right] .
$$

Here $\eta_{\sigma}(s)=\eta\left(\sigma^{-1} s\right)$, where $\eta$ is a smooth cut-off function such that

$$
\eta(s)=1, \text { for }|s|<1 \text { and } \eta(s)=0 \text { for }|s|>2,
$$

and $\sigma>0$ is a small $\varepsilon$-dependent number which for our purposes we take

$$
\sigma=\varepsilon^{\frac{1}{8}}
$$

The Laplacian operator in $\Omega_{\varepsilon}$ expressed in these new coordinates becomes

$$
\Delta_{y}=\Delta_{x, z}+B
$$

where $B$ is a second order differential operator with small coefficients:

$$
B=B_{22}(\varepsilon x, \varepsilon z) \frac{\partial^{2}}{\partial z^{2}}+B_{21}(\varepsilon x, \varepsilon z) \frac{\partial^{2}}{\partial x \partial z}+\varepsilon B_{20}(\varepsilon x, \varepsilon z) \frac{\partial}{\partial z},
$$

where $B_{i j}$ are smooth functions with $B_{22}(0, \cdot)=0, B_{21}(0, \cdot)=0$.

We also have, for $z=0$,

$$
\frac{\partial}{\partial \nu_{y}}=\frac{-1+\left|G_{0}^{\prime}(\varepsilon x)\right|^{2}}{\left(1+\left|G_{0}^{\prime}(\varepsilon x)\right|^{2}\right)^{1 / 2}} \frac{\partial}{\partial z}+\frac{G_{0}^{\prime}(\varepsilon x)}{\left(1+\left|G_{0}^{\prime}(\varepsilon x)\right|^{2}\right)^{1 / 2}} \frac{\partial}{\partial x},
$$

with a similar formula near $z=1 / \varepsilon$. Thus we write,

$$
\frac{\partial}{\partial \nu_{y}}=\frac{\partial}{\partial \nu}+B^{b}
$$

where

$$
B_{b}=b(\varepsilon x, \varepsilon z) \cdot \nabla_{x, z}, \quad l=0,1, \quad z=l / \varepsilon,
$$




$$
\frac{\partial}{\partial \nu}=(-1)^{\ell+1} \frac{\partial}{\partial z} \quad l=0,1, \quad z=l / \varepsilon
$$

Here $b$ is a smooth function with $b(0, \cdot)=0$. To define the approximate solution we recall that $U=U(s)$ is the heteroclinic solution to (1.7) such that $U( \pm \infty)= \pm 1$. More precisely we have:

$$
\begin{aligned}
U(s)-1 & =-A_{0} e^{-\sqrt{2} s}+o\left(e^{-\sqrt{2} s}\right), \quad \text { as } s \rightarrow+\infty, \\
U(s)+1 & =A_{0} e^{\sqrt{2} s}+o\left(e^{\sqrt{2} s}\right), \quad \text { as } s \rightarrow-\infty, \\
U^{\prime}(s) & =\sqrt{2} A_{0} e^{-\sqrt{2}|s|}+o\left(e^{-\sqrt{2}|s|}\right), \quad \text { as }|s| \rightarrow+\infty,
\end{aligned}
$$

where $A_{0}$ is a universal constant.

In the sequel we let $N>1$ be a fixed positive integer and consider $f_{1}, \ldots, f_{N}$ given, arbitrary functions $f_{k}:(0,1) \rightarrow \mathbb{R}$ which satisfy

$$
\left\|f_{k}\right\|_{H^{2}(0,1)} \leq|\log \varepsilon|^{3}, \quad f_{k+1}(\zeta)-f_{k}(\zeta) \geq \sqrt{2}|\log \varepsilon|-4 \sqrt{2} \log |\log \varepsilon| .
$$

For notational convenience we will also write

$$
f_{0}(\zeta)=-\delta_{0} / \varepsilon-f_{1}(\zeta) \text { and } f_{N+1}(\zeta)=\delta_{0} / \varepsilon-f_{N}(\zeta)
$$

Let us set

$$
w_{k}(x, z)=(-1)^{k+1} U\left(x-f_{k}(\varepsilon z)\right),
$$

and define the approximate solution to (2.1)-(2.2) by

$$
\mathrm{w}(x, z)=\sum_{k=1}^{N} w_{k}(x, z)+\frac{1}{2}\left((-1)^{N+1}-1\right),
$$

so that $\mathrm{w}(x, z) \sim w_{k}(x, z)$ for $\left|x-f_{k}(\varepsilon z)\right|$ not too large.

\subsection{Outline of the proof of Theorem 1.1.}

The approximation $\mathrm{w}$ defined by formula (3.1) does actually make sense in the infinite strip

$$
\mathcal{S}=\{(x, z) / 0<z<1 / \varepsilon\}
$$

Since the approximation $\mathrm{w}$ approaches its limits as $x \rightarrow \pm \infty$ at an exponential rate, and the remainder operators $B_{i}$ and $B_{b}$ are comparatively small, it is reasonable to believe that the solution of the full problem is essentially the same as that of the problem in the whole strip

$$
\Delta_{x, z} v+\eta_{\delta}^{\varepsilon} B v+f(v)=0 \quad \text { in } \mathcal{S}
$$

under boundary conditions

$$
\frac{\partial v}{\partial \nu}+\eta_{\delta}^{\varepsilon} B^{b} v=0 \quad \text { on } \partial \mathcal{S}
$$

Here and in what follows we denote

$$
\eta_{\delta}^{\varepsilon}(x, z):=\eta\left(\frac{\varepsilon|x|}{\delta}\right),
$$

where $\eta(s)$ is a smooth cut-off function as in (2.4), so that

$$
\eta_{\delta}^{\varepsilon}=1 \text { for }|x|<\frac{\delta}{\varepsilon}, \quad \eta_{\delta}^{\varepsilon}=0 \text { for }|x|>\frac{2 \delta}{\varepsilon} .
$$

In fact the full original problem in $\Omega_{\varepsilon}$ can be reduced to one in the strip by means of a gluing procedure developed in $\S 6$. 
We look for a solution of (2.7)-(2.8) in the form $w=\mathrm{w}+\phi$ where $\phi$ is globally small compared with $\mathrm{w}$. The equation for $\phi$ becomes

$$
\begin{gathered}
\Delta \phi+f^{\prime}(\mathrm{w}) \phi=-N(\phi)+E \quad \text { in } \mathcal{S} \\
\frac{\partial \phi}{\partial \nu}=-\eta_{\delta}^{\varepsilon} B^{b} \phi+E_{b} \quad \text { on } \partial \mathcal{S}
\end{gathered}
$$

where

$$
\begin{gathered}
N(\phi)=f(\mathrm{w}+\phi)-f(\mathrm{w})-f^{\prime}(\mathrm{w}) \phi+\eta_{\delta}^{\varepsilon} B \phi, \\
E=\Delta \mathrm{w}+\eta_{\delta}^{\varepsilon} B \mathrm{w}+f(\mathrm{w}), \quad E_{b}=\frac{\partial \mathrm{w}}{\partial \nu}+\eta_{\delta}^{\varepsilon} B^{b} \mathrm{w} .
\end{gathered}
$$

The operator $N(\phi)$ is the sum of a quadratic quantity in small $\phi$ and a small linear operator in $\phi$. The linear operator on the right hand side of equation (2.11) is also small. Thus if the linear operator defined by the left hand side of (2.10) were uniformly invertible in suitable norms under the associated Neumann boundary condition, one could recast the problem into a fixed point problem for a contraction mapping, provided of course that the errors $E$ and $E_{b}$ are small enough in the involved norms. However this uniform invertibility is not expected since there are decaying elements in an "approximate kernel" for the linear operator. Indeed, the functions $w_{j, x}=U_{x}\left(x-f_{j}(\varepsilon z)\right)$ "nearly anhilate" the operator, and unless the functions $f_{j}$ satisfy very special relations, we do not expect solvability of the original problem by the above means.

Thus we consider the linear operator

$$
L(\phi)=\Delta \phi+f^{\prime}(\mathrm{w}) \phi
$$

which we can rewrite as

$$
L(\phi)=\Delta-2 \phi+\left(f^{\prime}(\mathrm{w})+2\right) \phi=0 .
$$

Since $f^{\prime}(\mathrm{w})+2=O\left(e^{-\sqrt{2}|x|}\right)$ as $|x| \rightarrow \infty$, then the operator can be visualized as a small perturbation at infinity of $\Delta-2$. Standard elliptic regularity tells us that the problem

$$
\begin{aligned}
& \Delta \phi-2 \phi=h \quad \text { in } \mathcal{S}, \\
& \frac{\partial \phi}{\partial \nu}=g \quad \text { on }, \partial \mathcal{S}
\end{aligned}
$$

has the uniform a priori bound

$$
\|\phi\|_{H^{2}(\mathcal{S})} \leq C\left[\|h\|_{L^{2}(\mathcal{S})}+\|g\|_{H^{1}(\mathcal{S})}\right]
$$

and a corresponding existence statement. The analogous assertions for the operator $L$ does hold true for the following projected problem which "mods out" its above mentioned approximate kernel:

Given functions $h \in L^{2}(\mathcal{S}), g \in H^{1}(\mathcal{S})$, we consider the problem of finding $\phi \in H^{2}(\mathcal{S})$ such that for certain functions $c_{j} \in L^{2}(0,1), j=1, \ldots, N$ we have

$$
\begin{aligned}
& L(\phi)=h+\sum_{j=1}^{N} c_{j}(\varepsilon z) w_{j, x} \quad \text { in } \mathcal{S} \\
& \frac{\partial \phi}{\partial \nu}=g \quad \text { on } \partial \mathcal{S}, \\
& \int_{-\infty}^{\infty} \phi(x, z) w_{j, x}(x, z) d x=0, \quad \forall z \in\left(0, \frac{1}{\varepsilon}\right), j=1, \ldots, N .
\end{aligned}
$$


Proposition 2.1 There exists a constant $C>0$, independent of $\varepsilon$ and uniform for $f_{j}$ 's satisfying (2.5) such that for all small $\varepsilon$ Problem (2.14)-(2.16) has a solution $\phi=T(h, g)$, which defines a linear operator of its arguments and satisfies the estimate

$$
\|\phi\|_{H^{2}(\mathcal{S})} \leq C\left[\|h\|_{L^{2}(\mathcal{S})}+\|g\|_{H^{1}(\mathcal{S})}\right] .
$$

We will prove this proposition in $\S 3$. Using it, we would like to solve via contraction mapping principle the projected nonlinear problem

$$
\begin{aligned}
& L(\phi)=E-N(\phi)+\sum_{j=1}^{N} c_{j}(\varepsilon z) w_{j, x} \quad \text { in } \mathcal{S}, \\
& \frac{\partial \phi}{\partial \nu}=E_{b}-\eta_{\delta}^{\varepsilon} B_{b} \phi \quad \text { on } \partial \mathcal{S}, \\
& \int_{-\infty}^{\infty} \phi(x, z) w_{j, x}(x, z) d x=0, \quad j=1, \ldots, N .
\end{aligned}
$$

As we will see in $\S 4$, the inner error $E$ is essentially constituted by quantities carrying $\varepsilon^{2}|\log \varepsilon|^{q}$ times functions exponentially decaying in $x$ from the $f_{j}$ 's. This implies that

$$
\|E\|_{L^{2}(\mathcal{S})} \leq C \varepsilon^{\frac{3}{2}}|\log \varepsilon|^{q},
$$

for some $q>0$, uniformly on functions $f_{j}$ satisfying (2.5). This error however does not match in size with that at the boundary $E_{b}$ which is much larger: it only carries $\varepsilon$ rather than $\varepsilon^{2}$ as factors, so that we get

$$
\left\|E_{b}\right\|_{H^{1}(\mathcal{S})} \leq C \varepsilon^{\frac{1}{2}}|\log \varepsilon|^{q} .
$$

It is necessary to improve the approximation in order for both errors of approximation to share size, eliminating the terms of order $\varepsilon$ in the expansion of the boundary error. This is achieved in $\S 5$ by means of two steps: first, boundary conditions are imposed on the functions $f_{j}$. It is assumed that

$$
f_{k}^{\prime}(0)+\kappa\left(P_{0}\right) f_{k}(0)=0=f_{k}^{\prime}(1)-\kappa\left(P_{1}\right) f_{k}(1), \quad k=1, \ldots, N
$$

This assumption eliminates part of the $\varepsilon$-terms in $E_{b}$. Second, the remaining terms of order $\varepsilon$ are eliminated by building an improvement of approximation of the form $\mathrm{w}+\phi^{* *}$ where $\phi^{* *}$, of size $\varepsilon$, solves certain explicit linear problem and has the property to decay exponentially in $z$ direction towards the interior of $\mathcal{S}$. This procedure makes the initial problem $(2.17)-(2.19)$ equivalent to a similar one with new errors $E$ and $E_{b}$ with size $O\left(\varepsilon^{\frac{3}{2}}|\log \varepsilon|^{q}\right)$ and a qualitatively similar operator $N(\phi)$. By contraction mapping principle using Proposition 2.1, we then get a solution $\phi$ of this variation of (2.17)-(2.19) with

$$
\|\phi\|_{H^{2}(\mathcal{S})}=O\left(\varepsilon^{\frac{3}{2}}\right) .
$$

We shall get then a solution of our original problem if we are able to adjust the functions $f_{j}$ in such a way that

$$
c_{j}(z)=0 \quad \forall j=1, \ldots, N
$$

In order to solve the latter system of equations, we simply integrate equation (2.17) against $w_{j, x}$ to get

$$
c_{j}(z)=\int_{-\infty}^{\infty}\left(\Delta \mathrm{w}+\eta_{\delta}^{\varepsilon} B \mathrm{w}+f(\mathrm{w})\right) w_{x, j} d x+O\left(\varepsilon^{2+\mu}\right)
$$

for some $\mu>0$. These quantities are given at main order in the following lemma. 
Lemma 2.1 The following asymptotic formula holds

$$
\begin{gathered}
\int_{-\infty}^{\infty}\left(\Delta \mathrm{w}+\eta_{\delta}^{\varepsilon} B \mathrm{w}+f(\mathrm{w})\right) w_{x, k} d x= \\
(-1)^{k} a_{0}\left[\varepsilon^{2} b_{0} f_{k}^{\prime \prime}-e^{-\sqrt{2}\left(f_{k}-f_{k-1}\right)}+e^{-\sqrt{2}\left(f_{k+1}-f_{k}\right)}\right]+\mathcal{P}_{k},
\end{gathered}
$$

for some positive universal constants $a_{0}, b_{0}$, where

$$
\left\|\mathcal{P}_{k}\right\|_{L^{2}(0, \ell)} \leq C \varepsilon^{2+\mu}, \quad \text { for some } \mu>0,
$$

uniformly on functions $f_{k}$ 's satisfying conditions (2.5).

We carry our these computations in $\S 4$.

In this way, the full problem is reduced to solving a system of the form

$$
\varepsilon^{2} b_{0} f_{k}^{\prime \prime}-e^{-\sqrt{2}\left(f_{k}-f_{k-1}\right)}+e^{-\sqrt{2}\left(f_{k+1}-f_{k}\right)}=h,
$$

under boundary conditions (2.20), where $\|h\|_{L^{2}(0, \ell)}=O\left(\varepsilon^{2+\mu}\right)$ and $h$ itself is a nonlinear, nonlocal operator of $f$. The system for $h \equiv 0$ turns out to have a unique solution which is of size $O(|\log \varepsilon|)$. This solution is non-degenerate in a suitable sense, and the problem is finally solved via a perturbation argument. This part of the process is carried out in $\S 7$.

In the rest of this paper, we will work out in detail the above outlined scheme.

\section{Projected linear theory in the strip}

This section will be devoted to the resolution of the projected linear problem (2.14)-(2.16) by proving Proposition 2.1. A first claim we make is that to prove that result it suffices to consider the case $g=0$, so that we will only need to find the operator $T(h, 0)$. Indeed, let us consider the solution $\phi_{0}=\phi_{0}(g)$ to the problem

$$
\begin{aligned}
& \Delta \phi_{0}-\phi_{0}=0 \quad \text { in } \mathcal{S} \\
& \frac{\partial \phi_{0}}{\partial \nu}=g \quad \text { on } \partial \mathcal{S} .
\end{aligned}
$$

From standard elliptic theory, we find that

$$
\left\|\phi_{0}\right\|_{H^{2}(\mathcal{S})} \leq C\|g\|_{H^{1}(\mathcal{S})} .
$$

On the other hand, we check directly that

$$
\tilde{\phi}=\phi-\phi_{0}
$$

satisfies a similar equation, but now for $g=0$, with $\Lambda$ replaced by $\tilde{\Lambda}$, where

$$
\|\tilde{\Lambda}\|_{H^{2}(0,1 / \varepsilon)} \leq C\left[\|\Lambda\|_{H^{2}(0,1 / \varepsilon)}+\|g\|_{H^{1}(\mathcal{S})}\right],
$$

and with $h$ replaced by $\tilde{h}=\tilde{h}(h, g)$, a linear operator in its argument satisfying

$$
\|\tilde{h}\|_{L^{2}(\mathcal{S})} \leq C\left[\|h\|_{L^{2}(\mathcal{S})}+\|g\|_{H^{1}(\mathcal{S})}\right] .
$$

With the aid of this and the definition of $\tilde{\phi}$, the operator $T(h, g, \Lambda)$ is thus built just from $T(\tilde{h}, 0, \tilde{\Lambda})$, as claimed. 
For the proof of the proposition we need the validity of a priori estimates for a simpler problem. Given $h \in L^{2}(\mathcal{S})$, let us consider the operator

$$
L_{0}(\phi)=\Delta \phi+f^{\prime}(U(x)) \phi
$$

and the problem

$$
\begin{aligned}
& L_{0}(\phi)=h \quad \text { in } \mathcal{S}, \\
& \frac{\partial \phi}{\partial \nu}=0 \quad \text { on } \partial \mathcal{S} \\
& \int_{\mathbb{R}} \phi(x, z) U_{x}(x) d x=\Lambda(z), \quad 0<z<\frac{1}{\varepsilon} .
\end{aligned}
$$

where

$$
\|\Lambda\|_{H^{2}(0,1 / \varepsilon)} \leq C
$$

Lemma 3.1 There exists a constant $C>0$, independent of $\varepsilon$ such that solutions of (3.1)-(3.4) with $\Lambda$ satisfying (3.5) satisfy the a priori estimate

$$
\|\phi\|_{H^{2}(\mathcal{S})} \leq C\left[\|h\|_{L^{2}(\mathcal{S})}+\|\Lambda\|_{H^{2}(0,1 / \varepsilon)}\right] .
$$

\section{Proof.}

Assume first that $\Lambda \equiv 0$. Let us consider Fourier series decompositions for $h$ and $\phi$ of the form

$$
\begin{aligned}
& \phi(x, z)=\sum_{k=0}^{\infty} \phi_{k}(x) \cos (\pi k \varepsilon z) \\
& h(x, z)=\sum_{k=0}^{\infty} h_{k}(x) \cos (\pi k \varepsilon z) .
\end{aligned}
$$

Then we have the validity of the equations

$$
-k^{2} \varepsilon^{2} \phi_{k}+\mathcal{L}_{0}\left(\phi_{k}\right)=h_{k}, \quad x \in \mathbb{R}
$$

and conditions

$$
\int_{-\infty}^{\infty} \phi_{k} U_{x} d x=0
$$

for all $k$. We have denoted here

$$
\mathcal{L}_{0}\left(\phi_{k}\right)=\phi_{k, x x}+f^{\prime}(U(x)) \phi_{k} .
$$

Let us consider the bilinear form in $H^{1}(\mathbb{R})$ associated to the operator $\mathcal{L}_{0}$, namely

$$
B(\psi, \psi)=\int_{\mathbb{R}}\left[\left|\psi_{x}\right|^{2}-f^{\prime}(U)|\psi|^{2}\right] d x
$$

Since (3.7) holds uniformly in $k$ we conclude that

$$
C\left[\left\|\phi_{k}\right\|_{L^{2}(\mathbb{R})}^{2}+\left\|\phi_{k, x}\right\|_{L^{2}(\mathbb{R})}^{2}\right] \leq B\left(\phi_{k}, \phi_{k}\right)
$$

for a constant $C>0$ independent of $k$. Using this fact and equation (3.6) we find the estimate

$$
\left(1+k^{4} \varepsilon^{4}\right)\left\|\phi_{k}\right\|_{L^{2}(\mathbb{R})}^{2}+\left\|\phi_{k, x}\right\|_{L^{2}(\mathbb{R})}^{2} \leq C\left\|h_{k}\right\|_{L^{2}(\mathbb{R})}^{2} .
$$

In particular, we see from (3.6) that $\phi_{k}$ satisfies an equation of the form

$$
\phi_{k, x x}-2 \phi_{k}=\tilde{h}_{k}, \quad x \in \mathbb{R} .
$$


where $\left\|\tilde{h}_{k}\right\|_{L^{2}(\mathbb{R})} \leq C\left\|h_{k}\right\|_{L^{2}(\mathbb{R})}$. Hence it follows that additionally we have the estimate

$$
\left\|\phi_{k, x x}\right\|_{L^{2}(\mathbb{R})}^{2} \leq C\left\|h_{k}\right\|_{L^{2}(\mathbb{R})}^{2} .
$$

Adding up estimates (3.8), (3.9) in $k$ we conclude that

$$
\left\|D^{2} \phi\right\|_{L^{2}(\mathcal{S})}^{2}+\|D \phi\|_{L^{2}(\mathcal{S})}^{2}+\|\phi\|_{L^{2}(\mathcal{S})}^{2} \leq C\|h\|_{L^{2}(\mathcal{S})}^{2},
$$

which ends the proof in the case $\Lambda \equiv 0$. To prove the general case it suffices to apply the above argument with

$$
\tilde{\phi}=\phi-\Lambda(z) \frac{U_{x}(x)}{\int_{\mathbb{R}} U_{x}^{2}}
$$

We consider now the following problem: given $h \in L^{2}(\mathcal{S})$, find functions $\phi \in H^{2}(\mathcal{S}), c \in$ $L^{2}(0,1)$ such that

$$
\begin{aligned}
& L(\phi)=h+c(\varepsilon z) U_{x} \quad \text { in } \mathcal{S}, \\
& \frac{\partial \phi}{\partial \nu}=g \quad \text { on } \partial \mathcal{S}, \\
& \int_{\mathbb{R}} \phi U_{x} d x=\Lambda(z), \quad 0<z<\frac{1}{\varepsilon} .
\end{aligned}
$$

Lemma 3.2 Problem (3.10)-(3.12) possesses a unique solution

$$
\phi=T_{0}(h, g, \Lambda) .
$$

Moreover,

$$
\|\phi\|_{H^{2}(\mathcal{S})} \leq C\left[\|h\|_{L^{2}(\mathcal{S})}+\|\Lambda\|_{H^{2}(0,1 / \varepsilon)}+\|g\|_{H^{1}(\mathcal{S})}\right] .
$$

Proof. We first show that it is sufficient to prove this result for the case $\Lambda \equiv 0, g \equiv 0$. To this end let $\phi_{0}$ be the solution of

$$
\begin{aligned}
\Delta \phi_{0}-\phi_{0} & =0, \quad \text { in } \mathcal{S}, \\
\frac{\partial \phi_{0}}{\partial \nu} & =g, \quad \text { on } \partial \mathcal{S},
\end{aligned}
$$

and define

$$
\tilde{\phi}=\phi-\phi_{0}-\left[\Lambda(z)-\int_{\mathbb{R}} \phi_{0} U_{x} d x\right] \frac{U_{x}}{\left\|U_{x}\right\|_{L^{2}(\mathbb{R})}^{2}}
$$

Then $\tilde{\phi}$ satisfies $L(\tilde{\phi})=\tilde{h}+c(\varepsilon z) U_{x}$ with homogeneous boundary and orthogonality conditions and the general result will follow.

For existence, we write again

$$
h(x, z)=\sum_{k=0}^{\infty} h_{k}(x) \cos (\pi k \varepsilon z)
$$

and consider the problem of finding $\phi_{k} \in H^{1}(\mathbb{R})$, and constants $c_{k}$, such that

$$
-k^{2} \varepsilon^{2} \phi_{k}+\mathcal{L}_{0}\left(\phi_{k}\right)=h_{k}+c_{k} U_{x} \quad x \in \mathbb{R} .
$$

and

$$
\int_{\mathbb{R}} \phi_{k} U_{x} d x=0
$$


Fredholm's alternative yields that this problem is solvable with the choices

$$
c_{k}=-\frac{\int_{\mathbb{R}} h_{k} U_{x} d x}{\int_{\mathbb{R}} U_{x}^{2} d x} .
$$

Observe in particular that

$$
\sum_{k=0}^{\infty}\left|c_{k}\right|^{2} \leq C \varepsilon\|h\|_{L^{2}(\mathcal{S})}^{2}
$$

Finally define

$$
\phi(x, z)=\sum_{k=0}^{\infty} \phi_{k}(x) \cos (\pi k \varepsilon z),
$$

and correspondingly

$$
c(\zeta)=\sum_{k=0}^{\infty} c_{k} \cos (\pi k \zeta)
$$

Estimate $(3.13)$ gives that $c(\varepsilon z) U_{x}$ has its $L^{2}(\mathcal{S})$ norm controlled by that of $h$. The a priori estimates of the previous lemma tell us that the series for $\phi$ is convergent in $H^{2}(\mathcal{S})$ and defines a unique solution for the problem with the desired bounds.

In order to apply the previous result to the resolution of the full problem (2.14)-(2.16), we define first the operator

$$
L_{j}(\phi)=\Delta \phi+f^{\prime}\left(w_{j}\right) \phi
$$

and consider the following problem

$$
\begin{aligned}
& L_{j}(\phi)=h+c_{j}(\varepsilon z) w_{j, x} \text { in } \mathcal{S}, \\
& \frac{\partial \phi}{\partial \nu}=g \text { on } \partial \mathcal{S}, \\
& \int_{\mathbb{R}} \phi w_{j, x} d x=\Lambda(z) .
\end{aligned}
$$

We have

Lemma 3.3 Problem (3.14)-(3.16) possesses a unique solution

$$
\phi=T_{j}(h, g, \Lambda) .
$$

Moreover,

$$
\|\phi\|_{H^{2}(\mathcal{S})} \leq C\left[\|h\|_{L^{2}(\mathcal{S})}+\|\Lambda\|_{H^{2}(0,1 / \varepsilon)}+\|g\|_{H^{1}(\mathcal{S})}\right] .
$$

Proof. We recall that $w_{j}=(-1)^{j+1} U\left(x-f_{j}(\varepsilon z)\right)$. For a function $\xi(x, z)$ defined in $\mathcal{S}$ we denote below

$$
\tilde{\xi}(x, z)=\xi\left(x+f_{j}(\varepsilon z), z\right) .
$$

Direct computation gives that we see that Problem (3.14)-(3.16) is equivalent to

$$
\begin{aligned}
& \Delta \tilde{\phi}+B_{1}(\tilde{\phi})+f^{\prime}(U) \tilde{\phi}=\tilde{h}+c_{j}(\varepsilon z) U_{x} \text { in } \mathcal{S}, \\
& \frac{\partial \tilde{\phi}}{\partial \nu}=\tilde{g}+B_{2}(\tilde{\phi}) \quad \text { on } \partial \mathcal{S}, \\
& \int_{\mathbb{R}} \tilde{\phi} U_{x} d x=\Lambda(z),
\end{aligned}
$$

where

$$
\begin{aligned}
& B_{1}(\tilde{\phi})=\varepsilon^{2} f_{j}^{\prime}(\varepsilon z)^{2} \tilde{\phi}_{x x}+\varepsilon^{2} f_{j}^{\prime \prime}(\varepsilon z) \tilde{\phi}_{x}+2 \varepsilon f^{\prime}(\varepsilon z) \tilde{\phi}_{x z} \\
& B_{2}(\tilde{\phi})=\varepsilon f_{j}^{\prime}(\varepsilon z) \tilde{\phi}_{x}
\end{aligned}
$$


This problem is then equivalent to the fixed point linear problem

$$
\tilde{\phi}=T_{0}\left(\tilde{h}+B_{1}(\tilde{\phi}), \tilde{g}+B_{2}(\tilde{\phi}), \Lambda\right)
$$

where $T_{0}$ is the linear operator defined by Lemma 3.2. The linear operators $B_{1}$ and $B_{2}$ are small in the sense that

$$
\left\|B_{1}(\tilde{\phi})\right\|_{L^{2}(\mathcal{S})}+\left\|B_{2}(\tilde{\phi})\right\|_{H^{1}(\mathcal{S})} \leq o(1)\|\tilde{\phi}\|_{H^{2}(\mathcal{S})},
$$

with $o(1) \rightarrow 0$ as $\varepsilon \rightarrow 0$. From this, unique solvability of the problem and the desired estimate immediately follow.

Proof of Proposition 2.1. In order to solve for $\phi$ in Problem (2.14)-(2.16) we assume that $g=0$, which is sufficient as we have pointed out.

We search for a solution of $\phi=T(h, 0, \Lambda)$ in the form

$$
\phi=\sum_{j=1}^{N} \eta_{j} \phi+\psi
$$

where

$$
\eta_{j}(x, z)=\eta_{0}\left(\frac{x-f_{j}(\varepsilon z)}{R}\right), \quad R=\frac{\sqrt{2}}{2}|\log \varepsilon|,
$$

and $\eta_{0}$ is smooth with $\eta_{0}(s)=1$ for $|s|<1 / 2$ and $\eta_{0}(s)=0$ for $|s|>5 / 6$. We will denote

$$
\chi=1-\sum_{j=1}^{N} \eta_{j}
$$

It is readily checked that $\phi$ given by (3.17) solves Problem (2.14)-(2.16) with $g=0$ if the functions $\phi_{j}=\eta_{j} \phi, \psi$ satisfy the following linear system of equations.

$$
\begin{aligned}
\Delta \phi_{j}+f^{\prime}\left(w_{j}\right) \phi_{j}= & h \eta_{j}+c_{j}(\varepsilon z) w_{j, x} \\
& \quad-\left(f^{\prime}(\mathrm{w})-f^{\prime}\left(w_{j}\right)\right) \eta_{j} \phi_{j}-\eta_{j} f^{\prime}(\mathrm{w}) \psi \text { in } \mathcal{S}, \\
\frac{\partial \phi_{j}}{\partial \nu}= & 0 \text { on } \partial \mathcal{S} \\
\int_{\mathbb{R}} \phi_{j} w_{j, x} d x & =\tilde{\Lambda}_{j}
\end{aligned}
$$

where

$$
\Lambda_{j}=\int_{\mathbb{R}}\left(1-\eta_{j}\right) \phi_{j} w_{j, x} d x-\sum_{k \neq j} \int_{\mathbb{R}} \eta_{k} \phi_{k} w_{j, x} d x-\int_{\mathbb{R}} \psi w_{j, x}
$$

and

$$
\begin{aligned}
\Delta \psi+\chi f^{\prime}(\mathrm{w}) \psi= & \chi h+\sum_{j=1}^{N}\left(1-\eta_{j}\right) c_{j}(\varepsilon z) w_{j, x} \\
& -\sum_{j=1}^{N}\left[2 \nabla \eta_{j} \cdot \nabla \phi_{j}+\phi_{j} \Delta \eta_{j}\right] \\
\frac{\partial \psi}{\partial \nu}=0 & \text { on } \partial \mathcal{S},
\end{aligned}
$$

In order to solve this system we will set up a fixed point argument. To this end assume that function $\tilde{\phi}$ is given and define

$$
\tilde{\phi}_{j}=\tilde{\phi} \eta_{j}, \quad \tilde{\psi}=\tilde{\phi}-\sum_{j=1}^{N} \tilde{\phi}_{j}
$$


First we replace $\phi_{j}, \psi$ by $\tilde{\phi}_{j}, \tilde{\psi}$ on the right hand sides of (3.18), (3.20) and solve (3.18)-(3.20) for each $j=1, \ldots, n$ using Lemma 3.3. We get the following estimate

$$
\left\|\phi_{j}\right\|_{H^{2}(\mathcal{S})} \leq C\left[\|h\|_{L^{2}(\mathcal{S})}+\|\tilde{\psi}\|_{H^{2}(\mathcal{S})}+o(1) \sum_{j=1}^{N}\left\|\tilde{\phi}_{j}\right\|_{H^{2}(\mathcal{S})} .\right.
$$

Given $\tilde{\psi}$ we can now find functions $\phi_{j}=\phi_{j}(\tilde{\psi})$ which solve (3.18)-(3.20) by a fixed point argument. Next we observe that the norms $\left\|c_{j}(\varepsilon z) w_{j, x}\right\|_{L^{2}(\mathcal{S})}$ are controlled by $\|h\|_{L^{2}(\mathcal{S})}$ as it was pointed out in Lemma 3.2 (see (3.13) and the argument that follows). Therefore we can now solve (3.21)-(3.22) for $\psi$ which in addition satisfies

$$
\|\psi\|_{H^{2}(\mathcal{S})} \leq C\|h\|_{L^{2}(\mathcal{S})}+o(1) \sum_{j=1}^{N}\left\|\phi_{j}(\tilde{\psi})\right\|_{H^{2}(\mathcal{S})}
$$

Combining this with (3.23) and applying a fixed point argument again we get finally a solution to $(3.21)-(3.22)$. This ends the proof.

\section{The inner approximation: size and projections}

In what follows the error terms will often involve quantities of the type $O\left(\varepsilon^{p}|\log \varepsilon|^{q}\right)$. While keeping track of the powers of $\varepsilon$ is very important, the logarithmic factors turn out not to play a significant role. Therefore in the rest of the paper we will use $q$ to denote constant representing a power accompanying $|\log \varepsilon|$. The value of $q$ may change from line to line.

Our first goal is to compute the errors of approximation in a $\delta_{0} / \varepsilon$ neighborhood of $\Gamma_{\varepsilon}$, namely the quantities

$$
E_{0} \equiv \alpha(\mathrm{w})=\Delta_{y} \mathrm{w}+f(\mathrm{w}), \quad E_{0 b} \equiv \beta(\mathrm{w})=\frac{\partial \mathrm{w}}{\partial \nu_{y}} .
$$

We shall do this in $(x, z)$-coordinates.

The following result gives account of the size of the inner error of approximation $E_{0}$ in the region

$$
S_{\delta_{0} / \varepsilon}=\left\{|x|<\delta_{0} / \varepsilon, 0<z<1 / \varepsilon\right\},
$$

Lemma 4.1 The following estimate holds:

$$
\left|E_{0}(x, z)\right| \leq C \varepsilon^{2}\left(\left|f_{j}^{\prime \prime}(\varepsilon z)\right|+|\log \varepsilon|^{q}\right) \sum_{j=1}^{N} e^{-\sqrt{2}\left|x-f_{j}(\varepsilon z)\right|} \quad \text { in } S_{\delta_{0} / \varepsilon}
$$

uniformly on functions $f_{j}$ satisfying constraints (2.5). In particular

$$
\left\|E_{0}\right\|_{L^{2}\left(S_{\delta_{0} / \varepsilon}\right)} \leq C \varepsilon^{\frac{3}{2}}|\log \varepsilon|^{q} .
$$

Proof. We compute

$$
E_{0}=(\Delta+B) \mathrm{w}+f(\mathrm{w}) \equiv E_{01}+E_{02} .
$$

We have

$$
\begin{aligned}
\Delta \mathrm{w}=\sum_{k=1}^{N}( & -1)^{k+1} U^{\prime \prime}\left(x-f_{k}\right) \\
& -\varepsilon^{2} \sum_{k=1}^{N}(-1)^{k+1} f_{k}^{\prime \prime} U^{\prime}\left(x-f_{k}\right) \\
& +\varepsilon^{2} \sum_{k=1}^{N}(-1)^{k+1}\left(f_{k}^{\prime}\right)^{2} U^{\prime \prime}\left(x-f_{k}\right)
\end{aligned}
$$


Then, taking into account $B \mathrm{w}$ we get

$$
\begin{aligned}
\Delta \mathrm{w}+B \mathrm{w}=\sum_{k=1}^{N}( & -1)^{k+1} U^{\prime \prime}\left(x-f_{k}\right)\left[1-\varepsilon B_{21}(\varepsilon x, \varepsilon z) f_{k}^{\prime}\right] \\
& -\varepsilon^{2} \sum_{k=1}^{N}(-1)^{k+1} f_{k}^{\prime \prime} U^{\prime}\left(x-f_{k}\right)\left[1+B_{22}(\varepsilon x, \varepsilon z)\right] \\
& +\varepsilon^{2} \sum_{k=1}^{N}(-1)^{k+1}\left(f_{k}^{\prime}\right)^{2} U^{\prime \prime}\left(x-f_{k}\right)\left[1+B_{22}(\varepsilon x, \varepsilon z)\right] \\
& -\varepsilon^{2} B_{20}(\varepsilon x, \varepsilon z) \sum_{k=1}^{N}(-1)^{k+1} f_{k}^{\prime} U^{\prime}\left(x-f_{k}\right)
\end{aligned}
$$

We now turn to computing $E_{02}$. We fix a $k, 1 \leq k \leq N$ and consider the set

$$
A_{k}=\left\{(x, z) \mid \frac{f_{k-1}(\varepsilon z)+f_{k}(\varepsilon z)}{2} \leq x<\frac{f_{k+1}(\varepsilon z)+f_{k}(\varepsilon z)}{2}\right\} .
$$

For $x \in A_{k}$ we write

$$
\begin{gathered}
f(\mathrm{w})=f\left(w_{k}\right)+\left[f(\mathrm{w})-f\left(w_{k}\right)\right] \\
=f\left(w_{k}\right)+f^{\prime}\left(w_{k}\right)\left(\mathrm{w}-w_{k}\right)+\frac{1}{2} f^{\prime \prime}\left(w_{k}\right)\left(\mathrm{w}-w_{k}\right)^{2}+O\left(\left|\mathrm{w}-w_{k}\right|^{3}\right) \\
=f\left(w_{k}\right)+f^{\prime}\left(w_{k}\right)\left(\mathrm{w}-w_{k}\right)+\frac{1}{2} f^{\prime \prime}\left(w_{k}\right)\left(\mathrm{w}-w_{k}\right)^{2}+\max _{j \neq k} O\left(e^{-3 \sqrt{2}\left|f_{j}-x\right|}\right) \\
=\sum_{j=1}^{N} f\left(w_{j}\right)+\left[f^{\prime}\left(w_{k}\right)\left(\mathrm{w}-w_{k}\right)-\sum_{j \neq k} f\left(w_{j}\right)\right] \\
\quad+\frac{1}{2} f^{\prime \prime}\left(w_{k}\right)\left(\mathrm{w}-w_{k}\right)^{2}+\max _{j \neq k} O\left(e^{-3 \sqrt{2}\left|f_{j}-x\right|}\right) .
\end{gathered}
$$

It is convenient to introduce the following numbers.

$$
\sigma_{k j}= \begin{cases}(-1)^{j}, & \text { if } j<k \\ -(-1)^{j}, & \text { if } j>k\end{cases}
$$

Assuming that $N$ is odd we have

$$
\begin{gathered}
f^{\prime}\left(w_{k}\right)\left(\mathrm{w}-w_{k}\right)-\sum_{j \neq k} f\left(w_{j}\right)=\left[f^{\prime}\left(w_{k}\right)-f^{\prime}\left((-1)^{k+1}\right)\right]\left(\mathrm{w}-w_{k}\right) \\
+\left[f^{\prime}\left((-1)^{k+1}\right)\left(\mathrm{w}-w_{k}\right)-\sum_{j \neq k} f\left(w_{j}\right)\right] \\
=3\left[1-U^{2}\left(x-f_{k}(\varepsilon z)\right)\right]\left(\mathrm{w}-w_{k}\right)+f^{\prime}(1)\left\{\left(\mathrm{w}-w_{k}\right)-\sum_{j \neq k}\left[\sigma_{k j}-w_{j}\right]\right\} \\
\quad-\frac{1}{2} \sum_{j \neq k} f^{\prime \prime}\left(\sigma_{k j}\right)\left[\sigma_{k j}-w_{j}\right]^{2}+\max _{j \neq k} O\left(e^{-3 \sqrt{2}\left|f_{j}-x\right|}\right) \\
=\left\{3\left[1-U^{2}\left(x-f_{k}(\varepsilon z)\right)\right]\right\}\left(\mathrm{w}-w_{k}\right)-\frac{1}{2} \sum_{j \neq k} f^{\prime \prime}\left(\sigma_{k j}\right)\left[\sigma_{k j}-w_{j}\right]^{2} \\
+\max _{j \neq k} O\left(e^{-3 \sqrt{2}\left|f_{j}-x\right|}\right) .
\end{gathered}
$$


A similar argument applies when $N$ is even. Summarizing, we get

$$
\begin{gathered}
\left.E_{02}=\sum_{j=1}^{N} f\left(w_{j}\right)+\frac{1}{2} f^{\prime \prime}(\mathrm{w})\left(\mathrm{w}-w_{k}\right)^{2}+3\left[1-U^{2}\left(x-f_{k}\right)\right)\right]\left(\mathrm{w}-w_{k}\right) \\
-\sum_{j \neq k} \frac{1}{2} f^{\prime \prime}\left(\sigma_{k j}\right)\left(\sigma_{k j}-w_{j}\right)^{2}+\max _{j \neq k} O\left(e^{-3 \sqrt{2}\left|f_{j}-x\right|}\right)
\end{gathered}
$$

for $x \in A_{k}, k=1, \ldots, N$. It follows then that for $x \in A_{k}$,

$$
\begin{aligned}
E_{0}=-\varepsilon \sum_{k=1}^{N}(-1)^{k+1} f_{k}^{\prime} U^{\prime \prime}\left(x-f_{k}\right) B_{21}(\varepsilon x, \varepsilon z) \\
\quad-\varepsilon^{2} \sum_{k=1}^{N}(-1)^{k+1} f_{k}^{\prime \prime} U^{\prime}\left(x-f_{k}\right)\left[1+B_{22}(\varepsilon x, \varepsilon z)\right] \\
\quad+\varepsilon^{2} \sum_{k=1}^{N}(-1)^{k+1}\left(f_{k}^{\prime}\right)^{2} U^{\prime \prime}\left(x-f_{k}\right)\left[1+B_{22}(x, z)\right] \\
\quad-\varepsilon^{2} B_{2} \sum_{k=1}^{N}(-1)^{k+1} f_{k}^{\prime} U^{\prime}\left(x-f_{k}\right) \\
+\max _{j \neq k} O\left(e^{-2 \sqrt{2}\left|f_{j}-x\right|}\right) .
\end{aligned}
$$

Thus, denoting by $\chi_{A_{k}}(x)$ the characteristic function of the set $A_{k}$, we have

$$
E_{0}(x, z)=\sum_{k=1}^{N} \chi_{A_{k}}(x)\left[O\left(\varepsilon^{2}|\log \varepsilon|^{q}\right) e^{-\sqrt{2}\left|f_{k}-x\right|}+O(1) \max _{j \neq k} e^{-2 \sqrt{2}\left|f_{j}-x\right|}\right],
$$

and the result of the lemma readily follows.

As we have mentioned in the outline of the proof, it is of crucial importance the computation of the projections of the error against the functions $w_{j, x}(x, z)$ as given by Lemma 2.1. We carry out its proof next.

Proof of Lemma 2.1. Setting $\alpha(\mathrm{w})=\Delta \mathrm{w}+\eta_{\delta}^{\varepsilon} B \mathrm{w}+f(\mathrm{w})$, we have to compute

$$
\begin{aligned}
\int_{\mathbb{R}} \alpha(\mathrm{w}) U^{\prime}\left(x-f_{k}(\varepsilon z)\right) d x & =\left\{\int_{A_{k}}+\int_{\mathcal{S} \backslash A_{k}}\right\} \eta_{\delta}^{\varepsilon} \alpha(\mathrm{w}) U^{\prime}\left(x-f_{k}(\varepsilon z)\right) d x \\
& =\mathcal{E}_{k 1}(\varepsilon z)+\mathcal{E}_{k 2}(\varepsilon z) .
\end{aligned}
$$

We begin with

$$
\begin{aligned}
\mathcal{E}_{1 k}(\varepsilon z)=-\varepsilon^{2} & \int_{A_{k}} \sum_{j=1}^{N}(-1)^{j+1} f_{j}^{\prime \prime} U^{\prime}\left(x-f_{j}\right) U^{\prime}\left(x-f_{k}\right) \\
& +\varepsilon^{2} \int_{A_{k}} \sum_{j=1}^{N}(-1)^{j+1}\left(f_{j}^{\prime}\right)^{2} U^{\prime \prime}\left(x-f_{j}\right) U^{\prime}\left(x-f_{k}\right) \\
& +\int_{A_{k}}(B \mathrm{w}) U^{\prime}\left(x-f_{k}\right) \\
& +\int_{A_{k}}\left[E_{2}-\sum_{j=1}^{N} f\left(w_{j}\right)\right] U^{\prime}\left(x-f_{k}\right)=I_{1}+I_{2}+I_{3}+I_{4} .
\end{aligned}
$$


Using the asymptotic formula for $U^{\prime}$ we get

$$
I_{1}=(-1)^{k} \varepsilon^{2} f_{k}^{\prime \prime}(\varepsilon z) \int_{-\infty}^{\infty}\left(U^{\prime}(s)\right)^{2} d s+O\left(\varepsilon^{3}\right) \sum_{j=1}^{N} f_{j}^{\prime \prime}(\varepsilon z) .
$$

Using the fact that $\int_{-\infty}^{\infty} U^{\prime \prime}(s) U^{\prime}(s) d s=0$ we get

$$
I_{2}=O\left(\varepsilon^{3}\right) \sum_{j=1}^{N}\left(f_{j}^{\prime}(\varepsilon z)\right)^{2} .
$$

Now,

$$
I_{3}(\varepsilon z)=\varepsilon^{2} f_{k}^{\prime} b_{\sigma}(\varepsilon z) \int_{-\infty}^{\infty}\left(U^{\prime}(s)\right)^{2} d s+O\left(\sigma^{-2} \varepsilon^{3}\right) \sum_{j=1}^{N}\left[\left|f_{j}\right|^{\prime}+\left(f_{j}^{\prime}\right)^{2}+\left|f_{j}^{\prime \prime}\right|\right]
$$

where

$$
b_{\sigma}(\varepsilon z)=-\eta_{\sigma}(\varepsilon z) G_{0}^{\prime \prime}(0)-\eta_{\sigma}(1-\varepsilon z) G_{1}^{\prime \prime}(0) .
$$

Now we will evaluate $I_{4}$. Using the expressions of the error term $E_{2}$ found above we get

$$
\begin{aligned}
& E_{2}-\sum_{j=1}^{N} f\left(w_{j}\right)=\left[\frac{1}{2} f^{\prime \prime}(w)\left(w-w_{k}\right)^{2}-\frac{1}{2} \sum_{j \neq k} f^{\prime \prime}\left(\sigma_{k j}\right)\left(\sigma_{k j}-w_{j}\right)^{2}\right] \\
& +3\left(1-U^{2}\left(x-f_{k}\right)\right)\left(w-w_{k}\right) \equiv E_{21}+E_{22}
\end{aligned}
$$

We have

$$
\begin{aligned}
& E_{21}= \frac{1}{2} f^{\prime \prime}\left(w_{k}+\sum_{j \neq k}\left[\sigma_{k j}-w_{j}\right]\right)\left\{\sum_{j \neq k}\left[\sigma_{k j}-w_{j}\right]\right\}^{2} \\
& \quad-\frac{1}{2} \sum_{j \neq k} f^{\prime \prime}\left(\sigma_{k j}\right)\left[\sigma_{k j}-w_{j}\right]^{2} \\
&= \frac{1}{2} \sum_{j \neq k}\left[f^{\prime \prime}\left(w_{k}\right)-f^{\prime \prime}\left(\sigma_{k j}\right)\right]\left[\sigma_{k j}-w_{j}\right]^{2}+\max _{j \neq k} O\left(e^{-2 \sqrt{2}\left|f_{j}-f_{k}\right|}\right) \\
&=-3 \sum_{j \neq k}\left[w_{k}-\sigma_{k j}\right]\left[\sigma_{k j}-w_{j}\right]^{2}+\max _{j \neq k} O\left(e^{-2 \sqrt{2}\left|f_{j}-f_{k}\right|}\right) \\
&=-3(-1)^{k+1}\left[U\left(x-f_{k}\right)+1\right]\left[1-U\left(x-f_{k-1}\right)\right]^{2} \\
& \quad-3(-1)^{k+1}\left[U\left(x-f_{k}\right)-1\right]\left[1+U\left(x-f_{k+1}\right)\right]^{2} \\
& \quad+\varepsilon^{1 / 2} \max _{j \neq k} O\left(e^{-\sqrt{2}\left|f_{j}-f_{k}\right|}\right)
\end{aligned}
$$

For fixed $k$ let us consider the following integral

$$
\begin{aligned}
& \int_{\frac{f_{k-1}+f_{k}}{2}}^{f_{k}}\left[U\left(x-f_{k}\right)+1\right]\left[1-U\left(x-f_{k-1}\right)\right]^{2} U^{\prime}\left(x-f_{k}\right) d x \\
& \quad=\int_{\frac{f_{k-1}+f_{k}}{2}}^{f_{k}} O(1) e^{-2 \sqrt{2}\left(x-f_{k-1}\right)} e^{-2 \sqrt{2}\left(f_{k}-x\right)} d x \\
& \quad=O\left(e^{-2 \sqrt{2}\left(f_{k}-f_{k-1}\right)}\right)
\end{aligned}
$$

Similarly,

$$
\int_{f_{k}}^{\frac{f_{k+1}+f_{k}}{2}}\left[U\left(x-f_{k}\right)+1\right]\left[1-U\left(x-f_{k-1}\right)\right]^{2} U^{\prime}\left(x-f_{k}\right) d x=O\left(e^{-2 \sqrt{2}\left|f_{k-1}-f_{k}\right|}\right) .
$$


Therefore we get

$$
\int_{A_{k}} E_{21} U^{\prime}\left(x-f_{k}\right) d x=\varepsilon^{1 / 2} \max _{j \neq k} O\left(e^{-\sqrt{2}\left|f_{j}-f_{k}\right|}\right) .
$$

We will now compute

$$
\begin{gathered}
\int_{A_{k}} E_{22} U^{\prime}\left(x-f_{k}\right)= \\
3(-1)^{k+1} \int_{A_{k}}\left[1-U\left(x-f_{k}\right)^{2}\right]\left(1-U\left(x-f_{k-1}\right)\right) U^{\prime}\left(x-f_{k}\right) d x \\
-3(-1)^{k+1} \int_{A_{k}}\left[1-U\left(x-f_{k}\right)^{2}\right]\left(1+U\left(x-f_{k+1}\right)\right) U^{\prime}\left(x-f_{k}\right) \\
+\varepsilon^{1 / 2} \max _{j \neq k} O\left(e^{-\sqrt{2}\left|f_{j}-f_{k}\right|}\right) \\
=3(-1)^{k} A_{0} \int_{\mathbb{R}} a(s)\left[-e^{-\sqrt{2}\left(f_{k}-f_{k-1}\right)}+e^{-\sqrt{2}\left(f_{k+1}-f_{k}\right)}\right] \\
+\varepsilon^{1 / 2} \max _{j \neq k} O\left(e^{-\sqrt{2}\left|f_{j}-f_{k}\right|}\right),
\end{gathered}
$$

where we have denoted

$$
a(s)=\left(1-U^{2}(s)\right) U^{\prime}(s) e^{-\sqrt{2} s} .
$$

To compute $\mathcal{E}_{k 2}(\varepsilon z)$ we notice that for $x \in \mathcal{S} \backslash A_{k}$ we have $U^{\prime}\left(x-f_{k}\right)=\max _{j \neq k} O\left(e^{-\frac{\sqrt{2}}{2}\left|f_{j}-f_{k}\right|}\right)$ and thus we can estimate, using the above notation,

$$
\mathcal{E}_{k 2}(\varepsilon z)=\varepsilon^{1 / 2} \max _{j \neq k} O\left(e^{-\sqrt{2}\left|f_{j}-f_{k}\right|}\right)+O\left(\varepsilon^{1 / 2}\right) \sum_{m=1}^{4} I_{m}
$$

Now, let us define

$$
c_{1}=3 A_{0} \int_{-\infty}^{\infty}\left(1-U^{2}(s)\right) U^{\prime}(s) e^{-\sqrt{2} s} d s, \quad c_{0}=\int_{-\infty}^{\infty} U^{\prime}(s)^{2} d s .
$$

Gathering the above estimates, we get the following system for the approximate location of the interfaces:

$$
\begin{aligned}
\int_{\mathbb{R}} \alpha(\mathrm{w}) U^{\prime} & \left(x-f_{k}\right) d x= \\
(-1)^{k} & {\left[\varepsilon^{2} c_{0} f_{k}^{\prime \prime}(\varepsilon z)-c_{1}\left(e^{-\sqrt{2}\left(f_{k}-f_{k-1}\right)}-e^{-\sqrt{2}\left(f_{k+1}-f_{k}\right)}\right)\right] } \\
+\mathcal{P}_{k}(\varepsilon z), \quad k & =1, \ldots, N
\end{aligned}
$$

where, denoting $\zeta=\varepsilon z$, we have

$$
\begin{gathered}
\mathcal{P}_{k}(\zeta)=\varepsilon^{2} c_{0} f_{k}^{\prime} b_{\sigma}(\zeta)+O\left(\sigma^{-2} \varepsilon^{3}\right) \sum_{j=1}^{N}\left[\left|f_{j}^{\prime}\right|+\left|f_{j}^{\prime}\right|^{2}+\left|f_{j}^{\prime \prime}\right|\right] \\
+\varepsilon^{1 / 2} \max _{j \neq k} O\left(e^{-\sqrt{2}\left|f_{j}-f_{k}\right|}\right) .
\end{gathered}
$$

Now, we observe that

$$
\left\|\mathcal{P}_{k}\right\|_{L^{2}(0,1)} \leq C \varepsilon^{2+\mu}, \quad \text { for some } \mu>0, \quad k=1, \ldots, N
$$

This last estimate is possible thanks to the fact that we have chosen $\sigma=\varepsilon^{1 / 8}$. The proof is concluded. 


\section{$5 \quad$ Boundary error and improvement of approximation}

Next we compute the accuracy of the approximate solution w on the boundary. Again in $(x, z)$ coordinates we

$$
\begin{gathered}
E_{0 b}(z=0)=\frac{-1+\left|G_{0}^{\prime}(\varepsilon x)\right|^{2}}{\left(1+\left|G_{0}^{\prime}(\varepsilon x)\right|^{2}\right)^{1 / 2}} \frac{\partial \mathrm{w}}{\partial z}+\frac{G_{0}^{\prime}(\varepsilon x)}{\left(1+\left|G_{0}^{\prime}(\varepsilon x)\right|^{2}\right)^{1 / 2}} \frac{\partial \mathrm{w}}{\partial x} \\
=\varepsilon \sum_{j=1}^{N}\left[f_{j}^{\prime}(0)+G_{0}^{\prime \prime}(0) f_{j}(0)\right] \frac{\partial w_{j}}{\partial x} \\
+\varepsilon \sum_{j=1}^{N} G_{0}^{\prime \prime}\left(\varepsilon f_{j}(0)\right)\left(x-f_{j}(0)\right) \frac{\partial w_{j}}{\partial x} \\
+\varepsilon^{2} \sum_{j=1}^{N}\left[O\left(\left|x-f_{j}(0)\right|^{2}+O\left(\left|f_{j}(0)\right|^{2}\right)\right] \frac{\partial w_{j}}{\partial x}\right. \\
+\varepsilon^{3} \sum_{j=1}^{N}\left[O\left(\left|x-f_{j}(0)\right|^{2}+O\left(\left|f_{j}(0)\right|^{2}\right)\right] f_{j}^{\prime}(0) \frac{\partial w_{j}}{\partial x} .\right.
\end{gathered}
$$

A similar formula holds for $E_{0 b}(z=1 / \varepsilon)$. Thus we see that it is natural to take the following boundary conditions for $f_{j}$.

$$
\begin{aligned}
& f_{j}^{\prime}(0)+G_{0}^{\prime \prime}(0) f_{j}(0)=0 \\
& f_{j}^{\prime}(1)+G_{1}^{\prime \prime}(0) f_{j}(1)=0
\end{aligned}, \quad j=1, \ldots, N .
$$

We shall assume the validity of these conditions in the sequel. We observe that

$$
\left|E_{0 b}(z)\right| \leq C \varepsilon \sum_{k=1}^{N}\left|x-f_{k}(\varepsilon z)\right| e^{-\sqrt{2}\left|x-f_{k}(\varepsilon z)\right|} \quad \text { for } z=0, \varepsilon^{-1},
$$

which is one order of $\varepsilon$ worse in size than $E_{0}$. As we have said in the outline of the proof, the discrepancy between the order of approximation in the interior and on the boundary ( $E_{0}$ and $E_{0 b}$ respectively) makes it necessary to improve the original approximation w and eliminate the $O(\varepsilon)$-part of the error. We will construct an improvement in approximation by first solving the problem

$$
\begin{aligned}
\Delta \phi+f^{\prime}(U(x)) \phi & =0 \text { in } \mathcal{S} \\
\phi_{z}(x, 0) & =-x U_{x}, \quad \phi_{z}(x, 1 / \varepsilon)=0 \quad \text { on } \partial \mathcal{S} .
\end{aligned}
$$

We need the following result

Lemma 5.1 Problem (5.2)-(5.3) has a unique solution $\phi^{*} \in H^{2}(\mathcal{S})$ which is odd in $x$ for each z. Besides, there is a $C>0$ such that for all small $\varepsilon$,

$$
\left\|\phi^{*}\right\|_{H^{2}(\mathcal{S})} \leq C \text {. }
$$

In addition there exist constants $\nu<1 / 4, \mu>0$ and $C>0$ such that the following estimate holds:

$$
\left|\phi^{*}(x, z)\right|+\left|\nabla \phi^{*}(x, z)\right|+\left|D^{2} \phi^{*}(x, z)\right| \leq C e^{-[(1-\nu) \sqrt{2}|x|+\mu z]} .
$$

Proof. The existence part of the above lemma as well as estimate (5.4) follow from Lemma 3.2. Indeed, the problem

$$
\begin{array}{rlrl}
\Delta \phi+f^{\prime}(U(x)) \phi & =c(\varepsilon z) U_{x} \quad \text { in } \mathcal{S} & \\
\phi_{z}(x, 0) & =-x U_{x}, \quad \phi_{z}(x, 1 / \varepsilon)=0, \quad \text { on } \partial \mathcal{S} \\
\int_{\mathbb{R}} \phi U_{x} d x & =0 &
\end{array}
$$


has a unique solution $\phi^{*} \in H^{2}(S)$. On the other hand, the fact that $U_{x}(x)$ is an even function and uniqueness implies that $\phi$ is odd in $x$ for each $z$ and that $c \equiv 0$. Besides,

$$
\left\|\phi^{*}\right\|_{H^{2}(\mathcal{S})} \leq C\|g\|_{H^{1}(S)}
$$

where $g$ is any $H^{1}$-extension of the boundary condition. Let us take for instance

$$
g(x, z)=e^{-z} x U_{x}(x) \eta(2 \varepsilon z),
$$

with a suitable cutoff function $\eta$, in such a way that $\|g\|_{H^{1}(S)} \leq C$ with $C$ independent of $\varepsilon$. Thus we get

$$
\left\|\phi^{*}\right\|_{H^{2}(\mathcal{S})} \leq C,
$$

as desired.

Assuming now (5.4) we will established the decay estimates (5.5). We observe first that since $\phi^{*}$ is an odd function of $x$ therefore we have

$$
\int_{-\infty}^{\infty} \phi^{*}(x, z) U^{\prime}(x) d x=0
$$

hence

$$
\int_{-\infty}^{\infty}\left[\left|\phi_{x}^{*}(x, z)\right|^{2}-f^{\prime}(U)|\phi(x, z)|^{2}\right] d x \geq \mu_{2} \int_{-\infty}^{\infty}|\phi(x, z)|^{2} d x
$$

where $\mu_{2}$ is the second eigenvalue of the operator $L \psi=-\psi^{\prime \prime}-f^{\prime}(U) \psi$ considered in $\mathbb{R}$. It is known that $\mu_{2} \geq 2$. Consider function

$$
w(z)=\int_{-\infty}^{\infty}|\phi(x, z)|^{2} d x .
$$

From (5.9) it follows that

$$
-w_{z z}+\mu_{2} w \leq 0
$$

and from (5.4) we get that $\left|w_{z}(0)\right| \leq C$. Clearly we have also $w_{z}(1 / \varepsilon)=0$ and thus by a comparison argument we get that

$$
|w(z)| \leq C e^{-\mu z}, \quad \mu \leq \sqrt{2} .
$$

Using local elliptic estimates we then get

$$
\left|\phi^{*}(x, z) e^{\mu z}\right| \leq C, \quad \text { in } \mathcal{S} .
$$

From this, passing a suitable barrier we get (5.5).

We define the approximate solution of

$$
\begin{aligned}
\Delta \phi+f^{\prime}(\mathrm{w}) \phi & =0 \text { in } \mathcal{S} \\
\phi_{z}(x, l / \varepsilon) & =\varepsilon \sum_{j} G_{l}^{\prime \prime}(0)\left(x-f_{j}(l / \varepsilon)\right) \frac{\partial w_{j}}{\partial x} \quad \text { for } l=0,1 / \varepsilon
\end{aligned}
$$

by

$$
\phi^{* *}(x, z)=\varepsilon \sum_{j=1}^{N} \phi_{j}(x, z)
$$

where

$$
\phi_{j}(x, z)=\left[G_{0}^{\prime \prime}(0) \phi^{*}\left(x-f_{j}(\varepsilon z), z\right)+G_{1}^{\prime \prime}(0) \phi^{*}\left(x-f_{j}(\varepsilon z), \frac{1}{\varepsilon}-z\right)\right] .
$$

Our next goal is to prove the following 
Lemma 5.2 With the notation of the previous section we have the validity of the following fact

$$
\begin{gathered}
\left|\alpha\left(\mathrm{w}+\phi^{* *}\right)-\alpha(\mathrm{w})\right| \leq C \varepsilon^{3}\left(\left|f^{\prime \prime}(\varepsilon z)\right|+|\log \varepsilon|^{q}\right) \sum_{k=1}^{N} e^{-\sqrt{2}\left|f_{k}-x\right|} \\
+\varepsilon^{2} \sum_{j=1}^{N} e^{\left.-(1-\nu) \sqrt{2}\left|f_{j}-x\right|\right]}\left[e^{-\mu z}+e^{-\mu(1 / \varepsilon-z)}\right] .
\end{gathered}
$$

Consequently,

$$
\left\|\alpha\left(\mathrm{w}+\phi^{* *}\right)-\alpha(\mathrm{w})\right\|_{L^{2}(\mathcal{S})} \leq C \varepsilon^{\frac{3}{2}+\mu}, \quad \mu>0 .
$$

Similar decay estimates hold for $\beta\left(\mathrm{w}+\phi^{* *}\right)$. In addition there is an extension of $\beta\left(\mathrm{w}+\phi^{* *}\right)$ to the whole strip $\mathcal{S}$ such that

$$
\left\|\beta\left(\mathrm{w}+\phi^{* *}\right)\right\|_{H^{1}(\mathcal{S})} \leq C \varepsilon^{\frac{3}{2}}|\log \varepsilon|^{q} .
$$

Proof. We have

$$
\alpha\left(\mathrm{w}+\phi^{* *}\right)=E_{0}+\left[\left(\Delta+\eta_{\varepsilon}^{\delta} B\right) \phi^{* *}+f^{\prime}(\mathrm{w}) \phi^{* *}\right]+\mathcal{N}\left(\phi^{* *}\right)
$$

where

$$
\mathcal{N}\left(\phi^{* *}\right)=f\left(\mathrm{w}+\phi^{* *}\right)-f(\mathrm{w})-f^{\prime}(\mathrm{w}) \phi^{* *} .
$$

We fix a $k$ and consider the error in the set $A_{k}$, as in the previous section. We write

$$
\begin{aligned}
\Delta \phi^{* *}+f^{\prime}(\mathrm{w}) \phi^{* *}=\sum_{j=1}^{N} & {\left[\Delta \phi_{j}+f^{\prime}\left(w_{j}\right) \phi_{j}\right] } \\
& +\sum_{j=1}^{N} f^{\prime}\left(w_{j}\right)\left(\phi^{* *}-\phi_{j}\right) \\
& +\left[f^{\prime}\left(w_{k}\right)\left(\mathrm{w}-w_{k}\right)-\sum_{j \neq k} f^{\prime}\left(w_{j}\right)\right] \phi^{* *} \\
& +O(1)\left|\mathrm{w}-w_{k}\right|^{2} \phi^{* *} \\
= & \sum_{i=1}^{4} E_{1 i} .
\end{aligned}
$$

From the decay estimate (5.5) we get

$$
\left|E_{11}\right| \leq C \varepsilon^{3}|\log \varepsilon|^{q} e^{-(1-\nu) \sqrt{2}\left|f_{k}-x\right|},
$$

while the term $E_{12}$ is estimated using (5.5) by

$$
\left|E_{12}\right| \leq C \varepsilon^{2} \max _{j \neq k} e^{-(1-\nu) \sqrt{2}\left|f_{j}-x\right|}\left[e^{-\mu z}+e^{-\mu(1 / \varepsilon-z)}\right] .
$$

The remaining terms, including $B \phi^{* *}$ and $\mathcal{N}\left(\phi^{* *}\right)$ are easily seen to be smaller then the ones we have just considered. Estimate (5.10) follows immediately and (5.11) is an easy consequence of the construction. This ends the proof. 


\section{Reduction to a problem in $\mathcal{S}$}

We will now reduce the original problem which is defined in $\Omega_{\varepsilon}$ to a problem defined in the strip $\mathcal{S}$. This will be done using a gluing procedure similar to that in [12]. We consider smooth cut-off function $\eta(t)$ where $t \in \mathbb{R}$ such that $\eta(t)=1$ if $|t|<1 / 2$ and $=0$ if $|t|>1$.

Denote as well $\eta_{\delta}^{\varepsilon}(t)=\eta(\varepsilon t / \delta)$. We define our first global (i.e. defined in $\Omega_{\varepsilon}$ ) approximation to be simply

$$
\mathbf{w}(y)=\left\{\begin{array}{l}
\eta_{3 \delta}^{\varepsilon}\left(y_{1}\right)\left(\mathbf{w}+\phi^{* *}+1\right)-1, \text { if } y_{1}<0 \\
\eta_{3 \delta}^{\varepsilon}\left(y_{1}\right)\left(\mathbf{w}+\phi^{* *}-(-1)^{N+1}\right)+(-1)^{N+1}, \text { if } y_{1}>0
\end{array}\right.
$$

There is nothing wrong with considering $\delta$ as a quantity approaching zero with $\varepsilon$, however slowly. In fact we fix in the sequel

$$
\delta=\varepsilon^{\frac{1}{6}}
$$

We try a solution of $(2.1)-(2.2)$ of the form $v=\mathbf{w}+\tilde{\phi}$. Then

$$
\alpha(\mathbf{w}+\tilde{\phi})=0, \quad \beta(\mathbf{w}+\tilde{\phi})=0
$$

if and only if

$$
\begin{aligned}
\tilde{L}(\tilde{\phi}) & =\tilde{E}+\tilde{N}(\tilde{\phi}) \quad \text { in } \Omega_{\varepsilon} \\
\beta(\tilde{\phi}) & =\tilde{E}_{b} \quad \text { on } \partial \Omega_{\varepsilon}
\end{aligned}
$$

Here we have denoted

$$
\tilde{E}=\alpha(\mathbf{w}), \quad \tilde{L}(\tilde{\phi})=\Delta_{y} \tilde{\phi}+f^{\prime}(\mathbf{w}) \tilde{\phi}, \quad \tilde{E}_{b}=-\beta(\mathbf{w}),
$$

and

We further decompose $\tilde{\phi}$ in the following form:

$$
\tilde{N}(\tilde{\phi})=f(\mathbf{w}+\tilde{\phi})-f(\mathbf{w})-f^{\prime}(\mathbf{w}) \phi .
$$

$$
\tilde{\phi}=\eta_{3 \delta}^{\varepsilon} \phi+\psi
$$

where, in coordinates $(x, z)$, we assume that $\phi$ is defined in the whole strip $\mathcal{S}$, and with slight abuse of notation we call the same way its expression in $y$ coordinates and that in $(x, z)$. Substituting in (6.1) we find

$$
\tilde{L}\left(\eta_{3 \delta}^{\varepsilon} \phi\right)+\tilde{L}(\psi)=\tilde{E}+\tilde{N}\left(\eta_{3 \delta}^{\varepsilon} \phi+\psi\right) .
$$

We achieve this if the pair $(\phi, \psi)$ satisfies the following nonlinear coupled system:

$$
\begin{aligned}
\tilde{L}(\phi)=\eta_{\delta}^{\varepsilon} \tilde{E}+\eta_{\delta}^{\varepsilon} \tilde{N}(\phi+\psi)-\eta_{\delta}^{\varepsilon}\left[f^{\prime}(\mathbf{w})+2\right] \psi, & \text { in } \mathcal{S} \\
\frac{\partial \phi}{\partial \nu}+\eta_{3 \delta}^{\varepsilon} b \cdot \nabla_{x, z} \phi=-\eta_{\delta}^{\varepsilon} \tilde{E}_{b}, & \text { on } \partial \mathcal{S}
\end{aligned}
$$

and

$$
\begin{aligned}
& \Delta_{y} \psi-\left(2-2\left(1-\eta_{\delta}^{\varepsilon}\right)\left(2+f^{\prime}(\mathbf{w})\right)\right) \psi=\left(1-\eta_{\delta}^{\varepsilon}\right) \tilde{E}+2 \nabla \eta_{3 \delta}^{\varepsilon} \nabla \phi \\
& \quad+\left(\Delta \eta_{3 \delta}^{\varepsilon}\right) \phi+\left(1-\eta_{\delta}^{\varepsilon}\right) \tilde{N}\left(\eta_{3 \delta}^{\varepsilon} \phi+\psi\right) \\
& \frac{\partial \psi}{\partial \nu_{y}}=-\left(1-\eta_{\delta}^{\varepsilon}\right) \tilde{E}_{b}-\phi \frac{\partial \eta_{3 \delta}^{\varepsilon}}{\partial \nu_{y}}, \text { on } \partial \Omega_{\varepsilon}
\end{aligned}
$$

where $\phi$ is defined globally on $\mathcal{S}$ and $\psi$ is defined in $\Omega_{\varepsilon}$. Notice that the operator $\tilde{L}$ in the strip $\mathcal{S}$ may be taken as any compatible extension outside the $6 \delta / \varepsilon$-neighborhood of the curve. 
What we want to do next is to reduce the problem to one in the strip. To do this, we solve, given a small $\phi$, Problem (6.5)-(6.6) for $\psi$.

Since $1-\mathbf{w}^{2}=O\left(e^{-\gamma \delta / \varepsilon}\right)$ for $\left|y_{1}\right|>\delta \varepsilon^{-1}$, then the problem

$$
\begin{aligned}
\Delta_{y} \psi-2 \psi+\left(1-\eta_{\delta}^{\varepsilon}\right)\left(2+f^{\prime}(\mathbf{w})\right) \psi & =h, \text { in } \Omega_{\varepsilon} \\
\frac{\partial \psi}{\partial \nu_{y}} & =g \text { on } \partial \Omega_{\varepsilon}
\end{aligned}
$$

has a unique solution $\psi \in H^{2}\left(\Omega_{\varepsilon}\right)$ whenever $\|h\|_{L^{2}\left(\Omega_{\varepsilon}\right)},\|g\|_{H^{1}\left(\Omega_{\varepsilon}\right)}<+\infty$. Moreover,

$$
\|\psi\|_{L^{2}\left(\Omega_{\varepsilon}\right)} \leq C\left[\|h\|_{L^{2}\left(\Omega_{\varepsilon}\right)}+\|g\|_{H^{1}\left(\Omega_{\varepsilon}\right)}\right] .
$$

Assume now that $\phi \in H^{2}\left(\Omega_{\varepsilon}\right)$ satisfies that

$$
\|\phi\|_{H^{2}\left(\Omega_{\varepsilon}\right)} \leq C \varepsilon^{\frac{3}{2}} .
$$

Then from Sobolev's embedding

$$
\|\phi\|_{L^{\infty}\left(\Omega_{\varepsilon}\right)} \leq C \varepsilon
$$

Since $\tilde{N}$ has a power-like behavior with power greater than one, a direct application of contraction mapping principle yields that Problem (6.5)-(6.6) has a unique (small) solution $\psi=\psi(\phi)$ with

$$
\begin{aligned}
\|\psi(\phi)\|_{H^{2}\left(\Omega_{\varepsilon}\right) \leq} \leq & C\left[\left\|\left(1-\eta_{\delta}^{\varepsilon}\right) \tilde{E}\right\|_{L^{2}\left(\Omega_{\varepsilon}\right)}+\left\|\left(1-\eta_{\delta}^{\varepsilon}\right) \tilde{E}_{b}\right\|_{H^{1}\left(\Omega_{\varepsilon}\right)}\right] \\
& \left.+C \varepsilon \delta^{-1}\|\phi\|_{H^{2}\left(\Omega_{\varepsilon}\right)}\right]
\end{aligned}
$$

The nonlinear operator $\psi$ satisfies a Lipschitz condition of the form

$$
\left\|\psi\left(\phi_{1}\right)-\psi\left(\phi_{2}\right)\right\|_{H^{2}\left(\Omega_{\varepsilon}\right)} \leq C \varepsilon \delta^{-1}\left[\left\|\phi_{1}-\phi_{2}\right\|_{H^{2}\left(\Omega_{\varepsilon}\right)} .\right.
$$

The full problem has been reduced to solving the (nonlocal) problem in the infinite strip $\mathcal{S}$

$$
L_{2}(\phi)=\eta_{\delta}^{\varepsilon} \tilde{E}+\eta_{\delta}^{\varepsilon} \tilde{N}(\phi+\psi(\phi))-\eta_{\delta}^{\varepsilon}\left[f^{\prime}(\mathbf{w})+2\right] \psi(\phi)
$$

for a $\phi \in H^{2}(\mathcal{S})$ satisfying condition $(6.9)$. Here $L_{2}$ denotes a linear operator that coincides with $\tilde{L}$ on the region $|x|<\frac{6 \delta}{\varepsilon}$.

We shall define this operator next. The operator $\tilde{L}$ for $|x|<\frac{6 \delta}{\varepsilon}$ can be extended in coordinates $(x, z)$ to functions $\phi$ defined in the entire strip $\mathcal{S}$ as follows:

$$
L_{2}(\phi)=\Delta_{x, z} \phi+\eta_{6 \delta}^{\varepsilon} B(\phi)+f^{\prime}(\mathrm{w}) \phi=L(\phi)+\eta_{6 \delta}^{\varepsilon} B(\phi) .
$$

Rather than solving problem (6.3)-(6.4) directly, we shall do it in steps. We consider the following projected problem in $H^{2}(\mathcal{S})$ : given $\mathbf{f}=\left(f_{1}, \ldots, f_{N}\right)$ satisfying bounds $(2.5)$, find functions $\phi \in H^{2}(\mathcal{S})$, and $\mathbf{c}=\left(c_{1}, \ldots, c_{N}\right), c_{j} \in L^{2}(0,1)$ such that

$$
\begin{aligned}
& L(\phi)=\eta_{\delta}^{\varepsilon} \tilde{E}-N_{2}(\phi)+\sum_{j=1}^{N} c_{j}(\varepsilon z) w_{j, x} \text { in } \mathcal{S}, \\
& \frac{\partial \phi}{\partial \nu}+\eta_{3 \delta}^{\varepsilon} b \cdot \nabla_{x, z} \phi=-\eta_{\delta}^{\varepsilon} \tilde{E}_{b}, \quad \text { on } \partial \mathcal{S}, \\
& \int_{\mathbb{R}} \phi(x, z) w_{j, x}(x, z) d x=0,0<z<\frac{1}{\varepsilon}, j=1, \ldots, N .
\end{aligned}
$$

Here

$$
N_{2}(\phi)=\eta_{6 \delta}^{\varepsilon} B(\phi)+\eta_{\delta}^{\varepsilon} \tilde{N}(\phi+\psi(\phi))-\eta_{\delta}^{\varepsilon}\left[f^{\prime}(\mathbf{w})+2\right] \psi(\phi) .
$$


We will prove next that this problem has a unique solution whose norm is controlled by the $L^{2}$ norm of $\tilde{E}$ and $H^{1}$ of the suitable extension of $\tilde{E}_{b}$. After this has been done, our task is to adjust the parameter $\mathbf{f}$ in such a way that $\mathbf{c}$ is identically zero. As we will see, this turns out to be equivalent to solving a nonlocal, nonlinear second order differential equation for $\mathbf{f}$ Robin boundary conditions. As we will see this system is solvable in a region where the bound (2.5) hold.

\subsection{Solving the nonlinear intermediate problem}

Next we will solve problem (6.14)-(6.16).

Proposition 6.1 There exist numbers $D>0$ such that for all sufficiently small $\varepsilon$ and all $\mathbf{f}$ satisfying (2.5) problem (6.14)-(6.16) has a unique solution $\phi=\phi(\mathbf{f})$ which satisfies

$$
\|\phi\|_{H^{2}(\mathcal{S})} \leq D \varepsilon^{\frac{3}{2}}|\log \varepsilon|^{q},
$$

for certain $q$ depending on the accuracy of the approximation by the approximate solution $\mathbf{w}$ only. Besides $\phi$ depends continuously on $\mathbf{f}$.

Proof. Let $T_{\mathbf{f}}$ be the operator defined by Proposition 2.1, in which its dependence on the chosen $\mathbf{f}$ is emphasized. Let us denote

$$
M(\phi)=-\eta_{3 \delta}^{\varepsilon} b \cdot \nabla_{x, z} \phi
$$

Then, given $\mathbf{f}$ the equation (6.14)-(6.16) is equivalent to the fixed point problem for $\phi(\mathbf{f})$ :

$$
\phi(\mathbf{f})=T_{\mathbf{f}}\left(\eta_{\delta}^{\varepsilon} \tilde{E}(\mathbf{f})+N_{2}(\phi(\mathbf{f})),-\eta_{\delta}^{\varepsilon} \tilde{E}_{b}(\mathbf{f})+M(\phi(\mathbf{f})), 0\right) \equiv \mathcal{A}(\phi, \mathbf{f}) .
$$

In the sequel we will not emphasize the dependence on $\mathbf{f}$ whenever it is not necessary.

We will define now the region where contraction mapping principle applies. We consider the following closed, bounded subset of $H^{2}(\mathcal{S})$ :

$$
\mathcal{B}=\left\{\left.\phi \in H^{2}(\mathcal{S})\left|\|\phi\|_{H^{2}(\mathcal{S})} \leq D \varepsilon^{\frac{3}{2}}\right| \log \varepsilon\right|^{q},\right\}
$$

where $q>0$ is such that

$$
\|\tilde{E}\|_{L^{2}(\mathcal{S})}+\left\|\tilde{E}_{b}\right\|_{H^{1}(\mathcal{S})} \leq C \varepsilon^{3 / 2}|\log \varepsilon|^{q} .
$$

Recall that the existence of such $q$ has already been established in Lemma 5.2.

We claim that there is a constant $D>0$ such that the map $\mathcal{A}$ defined in (6.17) is a contraction from $\mathcal{B}$ into itself, uniform with respect to f. Given $\tilde{\phi} \in \mathcal{B}$ we denote $\phi=\mathcal{A}(\tilde{\phi}, \mathbf{f})$. First notice that using (6.10) and Lemma 5.2 we get for $\tilde{\phi} \in \mathcal{B}$

$$
\begin{aligned}
& \left\|\eta_{\delta}^{\varepsilon} \tilde{E}+N_{2}(\tilde{\phi})\right\|_{L^{2}(\mathcal{S})} \leq C_{0} \varepsilon^{3 / 2}|\log \varepsilon|^{q}+C\|\tilde{\phi}\|_{H^{2}(\mathcal{S})}^{2} \\
& +C \varepsilon^{1 / 4}\|\tilde{\phi}\|_{H^{2}} .
\end{aligned}
$$

Using Lemma 5.2, and the fact that $|b(\varepsilon x, \varepsilon z)| \leq C \varepsilon|x|$ we get for the $H^{1}$ extension of $-\eta_{\delta}^{\varepsilon} \tilde{E}_{b}+$ $M(\tilde{\phi})$ (denoted by the same symbol)

$$
\left\|-\eta_{\delta}^{\varepsilon} \tilde{E}_{b}+M(\tilde{\phi})\right\|_{H^{1}(\mathcal{S})} \leq C_{1} \varepsilon^{3 / 2}|\log \varepsilon|^{q}+C \delta\|\tilde{\phi}\|_{H^{2}(\mathcal{S})} .
$$

Since $\delta=\varepsilon^{1 / 6}$ from (6.19)-(6.20) we get that $\mathcal{A}$ indeed applies $\mathcal{B}$ into itself provided that $D$ is chosen sufficiently large. 
Next, let us analyze the Lipschitz character of the nonlinear operator involved in $\mathcal{A}$ for functions in $\mathcal{B}$, namely $N_{2}(\phi+\psi(\phi))$. For $\phi_{1}, \phi_{2} \in \mathcal{B}$ we have, using (6.10) and (6.11):

$$
\begin{gathered}
\left\|N_{2}\left(\phi_{1}+\psi\left(\phi_{1}\right)\right)-N_{2}\left(\phi_{2}+\psi\left(\phi_{2}\right)\right)\right\|_{L^{2}(\mathcal{S})} \leq \\
C \varepsilon^{3 / 2}|\log \varepsilon|^{q}\left\|\phi_{1}-\phi_{2}\right\|_{H^{2}(\mathcal{S})} .
\end{gathered}
$$

Using this, we readily find that $\mathcal{A}$ is a contraction map in $\mathcal{B}$ and thus show the existence of the fixed point.

A tedious but straightforward analysis of all terms involved in the differential operator and in the error yield that the operator $\mathcal{A}(\phi, \mathbf{f})$ is continuous with respect to $\mathbf{f}$. Indeed, denoting $\phi_{i}\left(\mathbf{f}_{i}\right)=\mathcal{A}\left(\tilde{\phi}_{i}, \mathbf{f}_{i}\right), i=1,2$, and indicating now the dependence on $\mathbf{f}_{i}$, let us write:

$$
\begin{aligned}
L_{2, \mathbf{f}_{1}}\left(\phi\left(\mathbf{f}_{1}\right)\right)-L_{2, \mathbf{f}_{2}}\left(\phi\left(\mathbf{f}_{2}\right)\right)= & L_{2, \mathbf{f}_{1}}\left[\phi\left(\mathbf{f}_{1}\right)-\phi\left(\mathbf{f}_{2}\right)\right] \\
& +\left[f^{\prime}\left(\mathbf{w}\left(\mathbf{f}_{1}\right)\right)-f^{\prime}\left(\mathbf{w}\left(\mathbf{f}_{2}\right)\right)\right] \phi\left(\mathbf{f}_{2}\right),
\end{aligned}
$$

and

$$
\begin{gathered}
\eta_{\delta}^{\varepsilon} \sum_{j=1}^{N}\left[c_{j}\left(\varepsilon z ; \mathbf{f}_{1}\right) w_{j, x}\left(\mathbf{f}_{1}\right)-c_{j}\left(\varepsilon z ; \mathbf{f}_{2}\right) w_{j, x}\left(\mathbf{f}_{2}\right)\right]= \\
\eta_{\delta}^{\varepsilon} \sum_{j=1}^{N}\left[c_{j}\left(\varepsilon z ; \mathbf{f}_{1}\right)-c_{j}\left(\varepsilon z ; \mathbf{f}_{2}\right)\right] w_{j, x}\left(\mathbf{f}_{1}\right) \\
\quad+\eta_{\delta}^{\varepsilon} \sum_{j=1}^{N} c_{j}\left(\varepsilon z ; \mathbf{f}_{2}\right)\left[w_{j, x}\left(\mathbf{f}_{1}\right)-w_{j, x}\left(\mathbf{f}_{2}\right)\right]
\end{gathered}
$$

and finally, for each $j=1, \ldots, N$

$$
\int_{\mathbb{R}}\left[\phi\left(\mathbf{f}_{1}\right)-\phi\left(\mathbf{f}_{2}\right)\right] w_{j, x}\left(\mathbf{f}_{1}\right) d x=-\int_{\mathbb{R}} \phi\left(\mathbf{f}_{2}\right)\left[\left[w_{j, x}\left(\mathbf{f}_{1}\right)-w_{j, x}\left(\mathbf{f}_{2}\right)\right] d x\right.
$$

Using this decomposition one can estimate $\left\|\phi\left(\mathbf{f}_{1}\right)-\phi\left(\mathbf{f}_{2}\right)\right\|_{H^{2}(\mathcal{S})}$ employing the theory developed in the previous section. Observe that this estimate does not depend on $c_{j}\left(\varepsilon z ; \mathbf{f}_{1}\right)-c_{j}\left(\varepsilon z ; \mathbf{f}_{2}\right)$. A rather lengthy but straightforward computation shows that in fact for fixed $\varepsilon$ the fixed point of $\mathcal{A}, \phi(\mathbf{f})$ is Lipschitz with respect to $\mathbf{f}$ :

$$
\left\|\phi\left(\mathbf{f}_{1}\right)-\phi\left(\mathbf{f}_{2}\right)\right\|_{H^{2}(\mathcal{S})} \leq C \varepsilon^{-1 / 2}|\log \varepsilon|^{q}\left\|\mathbf{f}_{1}-\mathbf{f}_{2}\right\|_{H^{2}(0,1)}
$$

and thus continuous with respect to $\mathbf{f}$. This ends the proof.

Clearly a solution to (6.14)-(6.16) will be a solution to (6.1)-(6.2), and consequently yield a solution to our original problem (2.1)-(2.2) if we can find $\mathbf{f}$ such that

$$
\mathbf{c}(\mathbf{f})=0 .
$$

As we will see this leads to a small perturbation of a system of $N$ nonlinear ODE's for $N$. We carry out this argument in the next section.

To prepare for this argument we will examine more closely the dependence of $\phi$ on $\mathbf{f}$. Notice first that the error term $\tilde{E}$ can be written in the form

$$
\tilde{E}(x, z ; \mathbf{f})=\tilde{E}_{1}\left(x, z ; \mathbf{f}, \mathbf{f}^{\prime}\right) \cdot\left(\varepsilon^{2} \mathbf{f}^{\prime \prime}\right)+\tilde{E}_{2}\left(x, z ; \mathbf{f}, \mathbf{f}^{\prime}\right),
$$


i.e. $\tilde{E}(x, z ; \mathbf{f})$ is actually linear as a function of $\varepsilon^{2} \mathbf{f}^{\prime \prime}$. Similarly the error term on the boundary $\beta(\mathbf{w})$ can be written as

$$
\beta(\mathbf{w})(\ell)=\beta(x ; \mathbf{f})(\ell)=O\left(\varepsilon^{2}\right) \beta_{1}(x ; \mathbf{f}(\ell))+O\left(\varepsilon^{2}\right) \beta_{2}(x ; \mathbf{f}(\ell)) \cdot\left(\varepsilon \mathbf{f}^{\prime}(\ell)\right),
$$

where $\ell=0,1 / \varepsilon$. Taking advantage of this structure of the error term we can refine estimates in Proposition 6.1 indicating their explicit dependence on $\mathbf{f}^{\prime \prime}$ as follows.

$$
\begin{aligned}
\|\phi(\mathbf{f})\|_{H^{2}(\mathcal{S})} \leq & C\left[\left\|\tilde{E}_{1}\left(\cdot ; \mathbf{f}, \mathbf{f}^{\prime}\right) \cdot\left(\varepsilon^{2} \mathbf{f}^{\prime \prime}\right)\right\|_{L^{2}(\mathcal{S})}+\left\|\beta_{2}(\cdot ; \mathbf{f}) \cdot\left(\varepsilon \mathbf{f}^{\prime}\right)\right\|_{H^{1}(\mathcal{S})}\right] \\
& +C\left[\left\|\tilde{E}_{2}\left(\cdot ; \mathbf{f}, \mathbf{f}^{\prime}\right)\right\|_{L^{2}(\mathcal{S})}+\left\|\beta_{1}(\cdot ; \mathbf{f})\right\|_{H^{1}(\mathcal{S})}\right] \\
\leq & D_{1} \varepsilon^{3 / 2}\left\|\mathbf{f}^{\prime \prime}\right\|_{L^{2}(0,1)}+D_{2} \varepsilon^{3 / 2}|\log \varepsilon|^{q}
\end{aligned}
$$

Exploring the linear dependence on $\varepsilon^{2} \mathbf{f}^{\prime \prime}$ further we can refine estimate (6.22) as follows

$$
\begin{aligned}
\left\|\phi\left(\mathbf{f}_{1}\right)-\phi\left(\mathbf{f}_{2}\right)\right\|_{H^{2}(\mathcal{S})} \leq C \varepsilon^{3 / 2}|\log \varepsilon|^{q}\left\|\mathbf{f}_{1}-\mathbf{f}_{2}\right\|_{H^{2}(0,1)} \\
+C \varepsilon^{-1 / 2}|\log \varepsilon|^{q}\left\|\mathbf{f}_{1}-\mathbf{f}_{2}\right\|_{H^{1}(0,1)}
\end{aligned}
$$

\section{Location of the interfaces and the Toda system}

\subsection{Location of the interfaces}

By integrating equation (6.14) against $w_{k, x}$ and integrating only in $x$ variable, we obtain that relations (6.23) are equivalent to the following system of equations for $\mathbf{f}$ :

$$
\int_{\mathbb{R}}\left[\eta_{\delta}^{\varepsilon} \tilde{E}+N_{2}(\phi)-L(\phi)\right] w_{k, x} d x=0, \quad k=1, \ldots, N
$$

We decompose on $|x|<\frac{6 \delta}{\varepsilon}$,

$$
\tilde{E}=\alpha(\mathrm{w})+\alpha\left(\mathrm{w}+\phi^{* *}\right)-\alpha(\mathrm{w})
$$

where

$$
\alpha(w)=\Delta w+\eta_{6 \delta}^{\varepsilon} B w+f(w)
$$

and recall that Lemma 2.1 gives us an expansion of the form

$$
\begin{gathered}
\int_{\mathbb{R}} \eta_{\delta}^{\varepsilon} \alpha(\mathrm{w}) U^{\prime}\left(x-f_{k}\right) d x= \\
(-1)^{k} a_{0}\left[\varepsilon^{2} b_{0} f_{k}^{\prime \prime}-e^{-\sqrt{2}\left(f_{k}-f_{k-1}\right)}+e^{-\sqrt{2}\left(f_{k+1}-f_{k}\right)}\right]+\mathcal{P}_{k}
\end{gathered}
$$

for some positive universal constants $a_{0}, b_{0}$, where

$$
\left\|\mathcal{P}_{k}\right\|_{L^{2}(0,1)} \leq C \varepsilon^{2+\mu}, \quad \text { for some } \mu>0,
$$

uniformly on functions $f_{k}$ 's satisfying conditions (2.5).

On the other hand, let us set

$$
\mathcal{R}_{k}(\varepsilon z)=-\int_{\mathbb{R}}\left(\alpha\left(\mathrm{w}+\phi^{* *}\right)-\alpha(\mathrm{w})\right) U^{\prime}\left(x-f_{k}\right) d x .
$$

Using Lemma 5.2 we get

$$
\mathcal{R}_{k}(\varepsilon z)=-\int_{\mathbb{R}}\left(\alpha\left(\mathrm{w}+\phi^{* *}\right)-\alpha(\mathrm{w})\right) U^{\prime}\left(x-f_{k}\right) d x
$$


that

$$
\left\|\mathcal{R}_{k}\right\|_{L^{2}(0,1)} \leq C \varepsilon^{2+\mu}
$$

Continuing with the terms involved in (7.1), using the contracting nature of $N_{2}(\phi)$ and Proposition 6.1 we get for

$$
\mathcal{Q}_{k}(\zeta)=\int_{\mathbb{R}} N_{2}(\phi) U^{\prime}\left(x-f_{k}\right) d x, \quad \zeta=\varepsilon z
$$

a similar estimate

$$
\left\|\mathcal{Q}_{k}\right\|_{L^{2}(0,1)} \leq C \varepsilon^{2+\mu}, \quad k=1, \ldots, N
$$

We should point out here that, by Proposition $6.1 \mathcal{Q}_{k}$ is a continuous function of $\mathbf{f}$.

Finally, with $\zeta=\varepsilon z$, we will write

$$
\begin{aligned}
\mathcal{S}_{k}(\zeta)= & \int_{\mathbb{R}} L_{2}(\phi) U^{\prime}\left(x-f_{k}\right) d x=\int_{\mathbb{R}} \phi_{z z} U^{\prime}\left(x-f_{k}\right) \\
& +\int_{\mathbb{R}} B(\phi) U^{\prime}\left(x-f_{k}\right) d x+\int_{\mathbb{R}} \phi\left[U_{x x}^{\prime}+f^{\prime}(\mathbf{w}) U^{\prime}\left(x-f_{k}\right)\right] d x .
\end{aligned}
$$

It is fairly straightforward to estimate $L^{2}$ norms of the functions involved in the definition of $\mathcal{S}_{k}$. For example, using the orthogonality conditions we can write

$$
\begin{aligned}
\mathcal{S}_{k}^{1}(\zeta)= & \int_{\mathbb{R}} \phi_{z z} U^{\prime}\left(x-f_{k}\right)=2 \varepsilon f_{k}^{\prime} \int_{\mathbb{R}} \phi_{z} U^{\prime \prime}\left(x-f_{k}\right) d x \\
& +\varepsilon^{2} \int_{\mathbb{R}} \phi\left[f_{k}^{\prime \prime} U^{\prime \prime}\left(x-f_{k}\right)-\left(f_{k}^{\prime}\right)^{2} U^{\prime \prime \prime}\left(x-f_{k}\right)\right] d x
\end{aligned}
$$

Using now Proposition 6.1 we get for $\left\|\mathcal{S}_{k}^{1}\right\|_{L^{2}(0,1)}$ an estimate similar to (7.5). As before it is also clear that $\mathcal{S}_{k}$ depends continuously on $\mathbf{f}$.

Let us define

$$
\mathcal{N}_{k}\left(\zeta ; \mathbf{f}, \mathbf{f}^{\prime}, \mathbf{f}^{\prime \prime}\right)=-(-1)^{k}\left[\mathcal{P}_{k}+\mathcal{Q}_{k}+\mathcal{R}_{k}-\mathcal{S}_{k}\right]
$$

where $\mathcal{P}_{k}$ is the quantity in expansion $(2.21)$.

Examining the formulas obtained above we see that $\mathcal{N}_{k}$ can be decomposed in the following way

$$
\mathcal{N}_{k}\left(\zeta ; \mathbf{f}, \mathbf{f}^{\prime}, \mathbf{f}^{\prime \prime}\right)=\mathcal{N}_{k 1}\left(\zeta ; \mathbf{f}, \mathbf{f}^{\prime}, \mathbf{f}^{\prime \prime}\right)+\mathcal{N}_{k 2}\left(\zeta ; \mathbf{f}, \mathbf{f}^{\prime}\right)
$$

where $\mathcal{N}_{k i}, i=1,2$ are continuous functions of its arguments. Function $\mathcal{N}_{k 1}$ satisfies in addition

$$
\begin{aligned}
& \left\|\mathcal{N}_{k 1}\left(\zeta ; \mathbf{f}, \mathbf{f}^{\prime}, \mathbf{f}^{\prime \prime}\right)\right\|_{L^{2}(0,1)} \leq C \varepsilon^{2+\mu} \\
& \left\|\mathcal{N}_{k 1}\left(\zeta ; \mathbf{f}_{1}, \mathbf{f}_{1}^{\prime}, \mathbf{f}_{2}^{\prime \prime}\right)-\mathcal{N}_{k 1}\left(\zeta ; \mathbf{f}_{2}, \mathbf{f}_{2}^{\prime}, \mathbf{f}_{2}^{\prime \prime}\right)\right\|_{L^{2}(0,1)} \leq \\
& C \varepsilon^{2+\mu}|\log \varepsilon|^{q}\left\|\mathbf{f}_{1}-\mathbf{f}_{2}\right\|_{H^{2}(0,1)},
\end{aligned}
$$

and the function $\mathcal{N}_{k 2}$ satisfies

$$
\left\|\mathcal{N}_{k 2}\left(\zeta ; \mathbf{f}, \mathbf{f}^{\prime}\right)\right\|_{L^{2}(0,1)} \leq C \varepsilon^{2+\mu}
$$

In addition the functions $f_{k}$ need to satisfy boundary conditions (5.1). Thus, after obvious algebra, setting $\theta=2(\zeta-1 / 2)$, and using expansion (2.21) in Lemma 2.1 we have the following system to deal with

$$
\begin{aligned}
\alpha_{0} \sqrt{2} \varepsilon^{2} f_{k}^{\prime \prime}-e^{-\sqrt{2}\left(f_{k}-f_{k-1}\right)}+e^{-\sqrt{2}\left(f_{k+1}-f_{k}\right)} & =\mathcal{N}_{k}, \\
& -1<\theta<1 \\
f_{k}^{\prime}(-1)+K_{-} f_{k}(-1) & =0, \\
f_{k}^{\prime}(1)+K_{+} f_{k}(1) & =0,
\end{aligned}
$$


where $K_{-}=\frac{1}{2} G_{0}^{\prime \prime}(0), K_{+}=\frac{1}{2} G_{1}^{\prime \prime}(0)$ and $\alpha_{0}=c_{0} /\left(c_{1} \sqrt{2}\right)$.

To solve the nonlinear system (7.9)-(7.11) we will first consider its version with $\mathcal{N}_{k}$ replaced by a given function $h_{k}$. Our goal is to prove the following:

Theorem 7.1 Assume that the following conditions hold:

$$
\begin{array}{r}
K_{-}>0, \quad K_{+}<0 \\
K_{-}-K_{+}+2 K_{-} K_{+}>0 \\
\frac{K_{-}+K_{+}}{2 K_{-} K_{+}} \in(-1,1)
\end{array}
$$

and let functions $h_{k}$ be such that

$$
\left\|h_{k}\right\|_{L^{2}(-1,1)} \leq C \varepsilon^{2+\mu}
$$

with some $\mu>0$. Then, for each sufficiently small $\varepsilon$ there exists a unique solution to the system (7.9)-(7.11) (with $h_{k}$ replacing $\mathcal{N}_{k}$ ) which satisfies

$$
\left\|f_{k}\right\|_{H^{2}(-1,1)} \leq C|\log \varepsilon|, \quad k=1, \ldots, N,
$$

and

$$
\begin{gathered}
f_{k+1}(\theta)-f_{k}(\theta)>\sqrt{2}|\log \varepsilon|-2 \sqrt{2} \log |\log \varepsilon|, \\
c>0, \quad k=1, \ldots, N-1 .
\end{gathered}
$$

Moreover, there exist $\bar{\lambda}, \bar{\lambda}=c|\log \varepsilon|+O(\log |\log \varepsilon|), \theta_{0} \in(0,1)$ such that we have the following representation

$$
\begin{aligned}
f_{k+1}(\theta)-f_{k}(\theta)= & \frac{1}{\sqrt{2}}\left[q_{k+1}\left(\bar{\lambda}\left(\theta-\theta_{0}\right)\right)-q_{k}\left(\bar{\lambda}\left(\theta-\theta_{0}\right)\right)-\log \bar{\lambda}^{2} \varepsilon^{2}\right] \\
& +\varphi_{k k+1}(\theta), \quad k=1, \ldots, N-1 .
\end{aligned}
$$

Functions $q_{k}$ are explicitly given solutions of the Toda system

$$
q_{k}^{\prime \prime}-e^{\left(q_{k-1}-q_{k}\right)}+e^{\left(q_{k}-q_{k+1}\right)}=0, \quad \text { in } \mathbb{R}, \quad k=1, \ldots, N-1
$$

where we take $q_{0}=-\infty, q_{N+1}=\infty$, and functions $\varphi_{k k+1}(\theta)$ satisfy

$$
\left\|\varphi_{k k+1}\right\|_{L^{2}} \leq C \varepsilon^{\mu^{\prime}}
$$

with some $\mu^{\prime}>0$.

We observe that since we have assumed $\left|\Gamma_{0}\right|=1$ and

$$
K_{-}=\frac{1}{2} \kappa\left(P_{0}\right), \quad K_{+}=-\frac{1}{2} \kappa\left(P_{1}\right),
$$

then the assumptions of Theorem 1.1 are precisely equivalent to (7.12)-(7.14).

In what follows we will first outline the proof of the theorem. Next we will prove a special case of two interfaces and then we will consider the general case. Finally we solve our nonlinear problem (7.9)-(7.11) thus concluding the proof of Theorem 1.1.

Let us set

$$
f_{k}=\frac{1}{\sqrt{2}}\left(\tilde{f}_{k}-(k-1) \log \left(\varepsilon^{2}\right)\right)
$$


so that (7.9)-(7.11) becomes

$$
\begin{aligned}
\alpha_{0} \tilde{f}_{k}^{\prime \prime}-e^{\left(\tilde{f}_{k-1}-\tilde{f}_{k}\right)}+e^{\left(\tilde{f}_{k}-\tilde{f}_{k+1}\right)} & =\tilde{h}_{k}, \quad-1<\theta<1, \\
\tilde{f}_{k}^{\prime}(-1)+K_{-} \tilde{f}_{k}(-1) & =K_{-}(k-1) \log \left(\varepsilon^{2}\right), \\
\tilde{f}_{k}^{\prime}(1)+K_{+} \tilde{f}_{k}(1) & =K_{+}(k-1) \log \left(\varepsilon^{2}\right),
\end{aligned}
$$

where $\tilde{h}_{k}=\varepsilon^{-2} h_{k}$. To solve the above problem will take advantage of the fact that some explicit solutions of the equations (7.15) considered on the whole real line are explicitly known. To this end we introduce two parameters $\theta_{0}$ and $|\lambda| \gg 1$, define a change of variables $t=\lambda \sqrt{\alpha_{0}}\left(\theta-\theta_{0}\right)$, and look for a solution to (7.15)-(7.17) in the form:

$$
\tilde{f}_{k}(\theta)=q_{k}\left(\bar{\lambda}\left(\theta-\theta_{0}\right)\right)-(k-1) \log \bar{\lambda}^{2}
$$

where for convenience we have set $\bar{\lambda}=\lambda \sqrt{\alpha_{0}}$. To solve

$$
\begin{aligned}
& q_{k}^{\prime \prime}-e^{\left(q_{k-1}-q_{k}\right)}+e^{\left(q_{k}-q_{k+1}\right)}=\bar{\lambda}^{-2} \tilde{h}_{k}, t^{-}<t<t^{+}, \\
& \bar{\lambda} q_{k}^{\prime}\left(t^{-}\right)+K_{-} q_{k}\left(t^{-}\right)=K_{-}(k-1) \log \left(\varepsilon^{2} \bar{\lambda}^{2}\right), \\
& \bar{\lambda} q_{k}^{\prime}\left(t^{+}\right)+K_{+} q_{k}\left(t^{+}\right)=K_{+}(k-1) \log \left(\varepsilon^{2} \bar{\lambda}^{2}\right),
\end{aligned}
$$

where $t^{-}=-\bar{\lambda}\left(1+\theta_{0}\right), t^{+}=\bar{\lambda}\left(1-\theta_{0}\right)$, we first solve (approximately) the homogeneous problem to determine just two parameters $\theta_{0}, \bar{\lambda}$ and next we need to determine the corrections $\phi_{k}$. This procedure will be described in detail in the sequel.

\subsection{Cluster of two interfaces}

In this section we will prove Theorem 7.1 in the special case when $N=2$. System (7.18)-(7.20) can then be reduced to a single scalar equation for $u=q_{1}-q_{2}$

$$
\begin{aligned}
u^{\prime \prime}+2 e^{u} & =\bar{\lambda}^{-2} h, \quad t^{-}<t<t^{+}, \\
\bar{\lambda} u^{\prime}\left(t^{-}\right)+K_{-} u\left(t^{-}\right) & =-K_{-} \log \left(\varepsilon^{2} \bar{\lambda}^{2}\right), \\
\bar{\lambda} u^{\prime}\left(t^{+}\right)+K_{+} u\left(t^{+}\right) & =-K_{+} \log \left(\varepsilon^{2} \bar{\lambda}^{2}\right) .
\end{aligned}
$$

The homogeneous version of the equation (7.21) considered on $\mathbb{R}$ has an explicit solution

$$
u_{0}(t)=\log \left(\frac{1}{4 \cosh ^{2}(t / 2)}\right)
$$

It can be seen easily that $u_{0}(t)<0$ and also that

$$
u_{0}(t)=-|t|+O\left(e^{-|t|}\right), \quad t \rightarrow \pm \infty .
$$

We will now look for the first approximation of the solution of (7.21)-(7.23) in the form $u(t)=$ $u_{0}(t)$. We get the following system for $\theta_{0}, \bar{\lambda}$ :

$$
\begin{aligned}
\bar{\lambda} u_{0}^{\prime}\left(-\bar{\lambda}\left(1+\theta_{0}\right)\right)+K_{-} u_{0}\left(-\bar{\lambda}\left(1+\theta_{0}\right)\right) & =-K_{-} \log \left(\varepsilon^{2} \bar{\lambda}^{2}\right), \\
\bar{\lambda} u_{0}^{\prime}\left(\bar{\lambda}\left(1-\theta_{0}\right)\right)+K_{+} u_{0}\left(\bar{\lambda}\left(1-\theta_{0}\right)\right) & =-K_{+} \log \left(\varepsilon^{2} \bar{\lambda}^{2}\right) .
\end{aligned}
$$

This is in fact a nonlinear system for $\left(\theta_{0}, \bar{\lambda}\right)$. Although it is in principle possible to find $\left(\theta_{0}, \bar{\lambda}\right)$ in such a way that (7.24)-(7.25) are satisfied exactly we will not do it here. Instead, taking into account the asymptotic behavior of $u_{0}$, we will look for $\left(\theta_{0}, \bar{\lambda}\right)$ that solve the following system

$$
\begin{aligned}
\bar{\lambda}\left[1-K_{-}\left(1+\theta_{0}\right)\right] & =-K_{-} \log \left(\varepsilon^{2} \bar{\lambda}^{2}\right) \\
\bar{\lambda}\left[-1-K_{+}\left(1-\theta_{0}\right)\right] & =-K_{+} \log \left(\varepsilon^{2} \bar{\lambda}^{2}\right)
\end{aligned}
$$


which has a solution $\left(\theta_{0}, \bar{\lambda}\right)$ such that $\bar{\lambda}=O(|\log \varepsilon|)>0, \theta_{0} \in(-1,1)$ thanks to (1.4) and our assumption. In fact we have that:

$$
\theta_{0}=\frac{K_{+}+K_{-}}{2 K_{+} K_{-}}, \quad \bar{\lambda}=\frac{K_{+} \log \left(\varepsilon^{2} \bar{\lambda}^{2}\right)}{1+K_{+}\left(1-\theta_{0}\right)}=\frac{K_{-} K_{+} \log \left(\varepsilon^{2} \bar{\lambda}^{2}\right)}{K_{-}-K_{+}+2 K_{-} K_{+}} .
$$

Notice that to find $\bar{\lambda}$ in (7.27) we have to solve a simple nonlinear equation. One can show that

$$
\bar{\lambda}=\frac{2 K_{+}}{-1+K_{+}\left(1-\theta_{0}\right)} \log \frac{1}{\varepsilon}+O\left(\log \log \frac{1}{\varepsilon}\right) .
$$

Now, denoting

$$
\bar{\lambda} u_{0}^{\prime}(t)+K_{ \pm} u_{0}(t)+K_{ \pm} \log \left(\epsilon^{2} \bar{\lambda}^{2}\right)=g_{ \pm}, \text {for } t=\mp \bar{\lambda}\left(1+ \pm \theta_{0}\right) \text {, respectively }
$$

we get from (7.28)

$$
\left|g_{ \pm}\right| \leq C \varepsilon^{\mu}
$$

with some $\mu>0$. We will seek an exact solution to (7.21)-(7.23) in the form

$$
u(t)=u_{0}(t)+\varphi(t) .
$$

To find $\varphi$ we will use a fixed point argument and thus we need to study the linearized version of $(7.21)-(7.23)$.

$$
\begin{aligned}
\phi^{\prime \prime}+2 e^{u_{0}} \phi & =h, \quad t^{-}<t<t^{+}, \\
\bar{\lambda} \phi^{\prime}\left(t^{-}\right)+K_{-} \phi\left(t^{-}\right) & =g_{-}, \\
\bar{\lambda} \phi^{\prime}\left(t^{+}\right)+K_{+} \phi\left(t^{+}\right) & =g_{+},
\end{aligned}
$$

with a given function $h \in L^{2}\left(t^{-}, t^{+}\right)$and constants $g_{ \pm}$. The functions

$$
\begin{aligned}
& \psi_{1}(t)=u_{0}^{\prime}(t), \\
& \psi_{2}(t)=t u_{0}^{\prime}(t)+2
\end{aligned}
$$

form the fundamental set for (7.30)-(7.32) and their Wronskian is actually $W\left(\psi_{1}, \psi_{2}\right)=1$. By the variation of constants formula,

$$
\begin{gathered}
\phi(t)=-\psi_{1}(t) \int_{-\bar{\lambda}\left(1+\theta_{0}\right)}^{t} \psi_{2}(s) h(s) d s+\psi_{2}(t) \int_{-\bar{\lambda}\left(1+\theta_{0}\right)}^{t} \psi_{1}(s) h(s) d s \\
+c_{1} \psi_{1}(t)+c_{2} \psi_{2}(t) .
\end{gathered}
$$

The functions $\psi_{1}, \psi_{2}$ satisfy the asymptotic formulas

$$
\psi_{1}(t)=\mp 1+O\left(e^{-|t|}\right), \quad \psi_{2}(t)=-|t|+O\left(e^{-|t|}\right), \quad t \rightarrow \pm \infty
$$

from which it follows easily

$$
\|\phi\|_{L^{2}} \leq C \bar{\lambda}^{3 / 2}\left[\|h\|_{L^{2}}+\bar{\lambda}^{-1}\left|c_{1}\right|+\left|c_{2}\right|\right] .
$$

To determine the constants $c_{1}, c_{2}$ we need to solve the system

$$
\begin{aligned}
c_{1}\left[\bar{\lambda} \psi_{1}^{\prime}+K_{-} \psi_{1}\right]+c_{2}\left[\bar{\lambda} \psi_{2}^{\prime}+K_{-} \psi_{2}\right]= & g_{-}, \quad \text { at } t=-\bar{\lambda}\left(1+\theta_{0}\right) \\
c_{1}\left[\bar{\lambda} \psi_{1}^{\prime}+K_{+} \psi_{1}\right]+c_{2}\left[\bar{\lambda} \psi_{2}^{\prime}+K_{+} \psi_{2}\right]= & g_{+} \\
& +K_{+}\left\{\psi_{1} \int_{-\bar{\lambda}\left(1+\theta_{0}\right)}^{\bar{\lambda}\left(1+\theta_{0}\right)} \psi_{2}(s) h(s) d s\right. \\
& \left.-\psi_{2} \int_{-\bar{\lambda}\left(1+\theta_{0}\right)}^{\bar{\lambda}\left(1+\theta_{0}\right)} \psi_{1}(s) h(s) d s\right\}, \\
& \text { at } t=\bar{\lambda}\left(1+\theta_{0}\right) .
\end{aligned}
$$


This system has a unique solution for $\bar{\lambda} \gg 1$ since the matrix

$$
\left(\begin{array}{cc}
K_{-} & 1-K_{-}\left(1+\theta_{0}\right) \\
-K_{+} & -1-K_{+}\left(1-\theta_{0}\right)
\end{array}\right)
$$

is nondegenerate thanks to the nondegeneracy condition (1.4). In fact we find

$$
\begin{aligned}
& \left|c_{1}\right| \leq C\left[\left|g_{-}\right|+\left|g_{+}\right|+\bar{\lambda}^{3 / 2}\|h\|_{L^{2}}\right] \\
& \left|c_{2}\right| \leq C \bar{\lambda}^{-1}\left[\left|g_{-}\right|+\left|g_{+}\right|+\bar{\lambda}^{3 / 2}\|h\|_{L^{2}}\right] .
\end{aligned}
$$

From (7.33) we get

$$
\|\phi\|_{L^{2}} \leq C\left[\bar{\lambda}^{2}\|h\|_{L^{2}}+\bar{\lambda}^{1 / 2}\left(\left|g_{-}\right|+\left|g_{+}\right|\right)\right] .
$$

By a straightforward argument we get a further estimate

$$
\|\phi\|_{\bar{\lambda}} \equiv\left\|\phi^{\prime \prime}\right\|_{L^{2}}+\left\|\phi^{\prime}\right\|_{L^{2}}+\|\phi\|_{L^{2}} \leq C\left[\bar{\lambda}^{2}\|h\|_{L^{2}}+\bar{\lambda}^{1 / 2}\left(\left|g_{-}\right|+\left|g_{+}\right|\right)\right] .
$$

Given (7.29) and assuming in addition that

$$
\|h\|_{L^{2}} \leq \varepsilon^{\mu},
$$

it is easy to solve (7.21)-(7.23) using a standard fixed point argument in the set of functions

$$
\mathcal{X}=\left\{\phi \mid\|\phi\|_{\bar{\lambda}}<\varepsilon^{\mu^{\prime}}\right\}, \quad \text { with } \mu^{\prime}<\mu .
$$

After this is done one can go back to the original problem solving the following equation for $f_{1}$

$$
f_{1}^{\prime \prime}=-\bar{\lambda}^{2} e^{u}+\varepsilon^{-2} h_{1}+O\left(e^{-c / \varepsilon}\right) e^{f_{1}}
$$

with the boundary conditions (7.10)-(7.11). The solution to this problem satisfies estimate $\left\|f_{1}\right\|_{H^{2}} \leq C|\log \varepsilon|$ as required. The rest of the Theorem 7.1 for $N=2$ follows now easily.

\subsection{Solvability theory for the general Toda system}

In this section we will prove Theorem 7.1. This will be done in several steps. We will assume here $N>2$ since the case $N=2$ has just been treated above.

For given functions $q_{j}(t), p_{j}(t), j=1, \ldots, N$ such that

$$
\sum_{j=1}^{N} q_{j}=\sum_{j=1}^{N} p_{j}=0,
$$

we define the Hamiltonian

$$
H=\sum_{j=1}^{N} \frac{p_{j}^{2}}{2}+V, \quad V=\sum_{j=1}^{N-1} e^{\left(q_{j}-q_{j+1}\right)}
$$

From what we have seen above (4.27), to determine the location of the interfaces it is necessary to develop a solvability theory for a Toda system of the form:

$$
\begin{aligned}
\frac{d q_{j}}{d t} & =p_{j} \\
\frac{d p_{j}}{d t} & =-\frac{\partial H}{\partial q_{j}} .
\end{aligned}
$$


In this section we will often make use of classical results of Konstant [23] and in particular we will use the explicit formula for the solutions of (7.38)-(7.39) (see formula (7.7.10) in [23]). We will first introduce some notation. Given numbers $w_{1}, \ldots, w_{N} \in \mathbb{R}$ such that

$$
\sum_{k=1}^{N} w_{k}=0, \quad \text { and } w_{k}>w_{k+1}, \quad k=1, \ldots, N
$$

we define

$$
\mathbf{w}_{0}=\operatorname{diag}\left(w_{1}, \ldots, w_{N}\right) .
$$

Furthermore, given numbers $g_{1}, \ldots, g_{n} \in \mathbb{R}$ such that

$$
\prod_{k=1}^{N} g_{k}=1, \quad \text { and } g_{k}>0, \quad k=1, \ldots, N
$$

we define

$$
\mathbf{g}_{0}=\operatorname{diag}\left(g_{1}, \ldots, g_{N}\right) .
$$

The matrices $\mathbf{w}_{0}$ and $\mathbf{g}_{0}$ can be parametrized by introducing the following two sets of parameters

$$
c_{k}=w_{k}-w_{k+1}, d_{k}=\log g_{k+1}-\log g_{k}, k=1, \ldots, N-1 .
$$

We define functions $\Phi_{k}\left(\mathbf{g}_{0}, \mathbf{w}_{0} ; t\right), t \in \mathbb{R}, k=0, \ldots, N$, by

$$
\begin{aligned}
& \Phi_{0}=\Phi_{N} \equiv 1 \\
& \Phi_{k}\left(\mathbf{g}_{0}, \mathbf{w}_{0} ; t\right)= \\
& (-1)^{k(n-k)} \sum_{1 \leq i_{i}<\cdots<i_{k} \leq N} r_{i_{1} \ldots i_{k}}\left(\mathbf{w}_{0}\right) g_{i_{1}} \ldots g_{i_{k}} \exp \left[-t\left(w_{i_{1}}+\cdots+w_{i_{k}}\right)\right]
\end{aligned}
$$

where $r_{i_{1} \ldots i_{k}}\left(\mathbf{w}_{0}\right)$ are rational functions of the entries of the matrix $\mathbf{w}_{0}$. It is proven in [23] that all solutions of (7.38)-(7.39) are of the form

$$
q_{j}(t)=\log \Phi_{j-1}\left(\mathbf{g}_{0}, \mathbf{w}_{0} ; t\right)-\log \Phi_{j}\left(\mathbf{g}_{0}, \mathbf{w}_{0} ; t\right), j=1, \ldots, N
$$

We introduce variables $u_{k}=q_{k}-q_{k+1}$. In terms of $\mathbf{u}=\left(u_{1}, \ldots, u_{N-1}\right)$ system (7.38)-(7.39) becomes

$$
\mathbf{u}^{\prime \prime}+M e^{\mathbf{u}}=0,
$$

where

$$
M=\left(\begin{array}{cccc}
2 & -1 & 0 \cdots & 0 \\
-1 & 2 & -1 \cdots & 0 \\
& & \ddots & \\
0 & \cdots & 2 & -1 \\
0 & \cdots & -1 & 2
\end{array}\right), \quad e^{\mathbf{u}}=\left(\begin{array}{c}
e^{u_{1}} \\
\vdots \\
e^{u_{N-1}}
\end{array}\right)
$$

As a consequence of (7.41) all solutions to (7.43) are given by

$$
\begin{aligned}
u_{j}(t)=q_{j}(t)-q_{j+1}(t)=- & 2 \log \Phi_{j}\left(\mathbf{g}_{0}, \mathbf{w}_{0} ; t\right)+\log \Phi_{j-1}\left(\mathbf{g}_{0}, \mathbf{w}_{0} ; t\right) \\
& +\log \Phi_{j+1}\left(\mathbf{g}_{0}, \mathbf{w}_{0} ; t\right)
\end{aligned}
$$

Our first goal is to prove the following:

Lemma 7.1 Let $\mathbf{w}_{0}$ be such that

$$
\left|w_{k}-(N-k)+\frac{N-1}{2}\right|<\frac{1}{100 N^{4}}, \quad k=1, \ldots, N .
$$


Then there holds

$$
u_{k}(t)=\left\{\begin{array}{l}
-c_{N-k} t-d_{N-k}+\tau_{k}^{+}(\mathbf{c})+O\left(e^{-\frac{|t|}{2}}\right), \text { as } t \rightarrow+\infty, \quad k=1, \ldots, N-1 \\
c_{k} t+d_{k}+\tau_{k}^{-}(\mathbf{c})+O\left(e^{-\frac{|t|}{2}}\right), \text { as } t \rightarrow-\infty, \quad k=1, \ldots, N-1
\end{array}\right.
$$

where $\tau_{k}^{ \pm}(\mathbf{c})$ are smooth functions of the vector $\mathbf{c}=\left(c_{1}, \ldots, c_{N-1}\right)$.

Proof. Let $q_{j}, j=1, \ldots, N$ be a solution of the system (7.38)-(7.39) depending on the (matrix valued) parameters $\mathbf{w}_{0}, \mathbf{g}_{0}$ and defined in (7.42). We need to study the asymptotic behavior of $\Phi_{j}\left(\mathbf{w}_{0}, \mathbf{g}_{0} ; t\right)$ as $t \rightarrow \pm \infty$ with the entries of $\mathbf{w}_{0}$ satisfying (7.45) and still undetermined $\mathbf{g}_{0}$.

Note that inequalities (7.45) together with the condition $\sum_{k=1}^{N} w_{k}=0$ imply

$$
\left|c_{k}-1\right|<\frac{1}{50 N^{3}}, \quad k=1, \ldots, N
$$

By (7.41) and (7.47), we get that as $t \rightarrow-\infty$

$$
\Phi_{k}=(-1)^{k(N-k)} r_{1 \ldots k}\left(\mathbf{w}_{0}\right) g_{1} \ldots g_{k} e^{-\left(w_{1}+\cdots+w_{k}\right) t}+O\left(e^{-|t|(1+k(N-k) / 4)}\right),
$$

hence

$$
\frac{\Phi_{k+1} \Phi_{k-1}}{\Phi_{k}^{2}}=\frac{g_{k+1} r_{1 \ldots(k-1)}\left(\mathbf{w}_{0}\right) r_{1 \ldots(k+1)}\left(\mathbf{w}_{0}\right) e^{-t}}{g_{k} r_{1 \ldots k}^{2}\left(\mathbf{w}_{0}\right)}\left(1+O\left(e^{-\frac{|t|}{2}}\right)\right) .
$$

It follows that as $t \rightarrow-\infty$

$$
\begin{aligned}
u_{k}(t) & =\log \left(\frac{\Phi_{k+1} \Phi_{k-1}}{\Phi_{k}^{2}}\right) \\
& =-c_{k} t+\log \left(\frac{g_{k+1} r_{1 \ldots(k-1)}\left(\mathbf{w}_{0}\right) r_{1 \ldots(k+1)}\left(\mathbf{w}_{0}\right)}{g_{k} r_{1 \ldots k}^{2}\left(\mathbf{w}_{0}\right)}\right)+O\left(e^{-\frac{|t|}{2}}\right) \\
& =c_{k} t+d_{k}+\tau_{k}^{-}(\mathbf{c})+O\left(e^{-\frac{|t|}{2}}\right)
\end{aligned}
$$

where

$$
\tau_{k}^{-}(\mathbf{c})=\log \left(\frac{r_{1 \ldots(k-1)}\left(\mathbf{w}_{0}\right) r_{1 \ldots(k+1)}\left(\mathbf{w}_{0}\right)}{r_{1 \ldots k}^{2}\left(\mathbf{w}_{0}\right)}\right) .
$$

Similarly, as $t \rightarrow+\infty$ we get

$$
\begin{aligned}
& u_{k}(t)=\log \left(\frac{\Phi_{k+1} \Phi_{k-1}}{\Phi_{k}^{2}}\right) \\
& =-c_{N-k} t-d_{N-k}+\tau_{k}^{+}+O\left(e^{-\frac{|t|}{2}}\right)
\end{aligned}
$$

where

This ends the proof.

$$
\tau_{k}^{+}(\mathbf{c})=\log \left(\frac{r_{N+2-k \ldots N}\left(\mathbf{w}_{0}\right) r_{N-k \ldots N}\left(\mathbf{w}_{0}\right)}{r_{N+1-k \ldots N}^{2}\left(\mathbf{w}_{0}\right)}\right) .
$$

Next we need to choose $\left(c_{k}, d_{k}\right)$ : let $\left(\bar{\lambda}, \theta_{0}\right)$ be defined at $(7.26)$ as in the case of $N=2$ and

$$
c_{k}=1+\frac{1}{\bar{\lambda}} \bar{c}_{k}
$$

We need to choose $u_{k}$ such that it satisfies

$$
\begin{aligned}
& \bar{\lambda} u_{k}^{\prime}\left(t^{-}\right)+K_{-} u_{k}\left(t^{-}\right)=K_{-} \log \left(\varepsilon^{2} \bar{\lambda}^{2}\right)+O\left(\varepsilon^{\mu}\right) \\
& \bar{\lambda} u_{k}^{\prime}\left(t^{+}\right)+K_{+} u_{k}\left(t^{+}\right)=K_{+} \log \left(\varepsilon^{2} \bar{\lambda}^{2}\right)+O\left(\varepsilon^{\mu}\right)
\end{aligned}
$$


for some $\mu>0$. To this end, we set $\left(\hat{c}_{k}, d_{k}\right)$ such that

$$
\begin{aligned}
& -\left(1+K_{+}\left(1-\theta_{0}\right)\right) \hat{c}_{N-k}-K_{+} d_{N-k}+K_{+} \tau_{k}^{+}\left(\mathbf{c}_{0}+\frac{1}{\bar{\lambda}} \hat{\mathbf{c}}\right)=0 \\
& \left(1-K_{-}\left(1+\theta_{0}\right)\right) \hat{c}_{k}+K_{-} d_{k}+K_{-} \tau_{k}^{-}\left(\mathbf{c}_{0}+\frac{1}{\bar{\lambda}} \hat{c}\right)=0
\end{aligned}
$$

where $\mathbf{c}_{0}=(1, \ldots, 1), \hat{\mathbf{c}}=\left(\hat{c}_{1}, \ldots, \hat{c}_{N-1}\right)$.

By changing $k$ to $N-k$ in (7.55), we see that (7.55)-(7.56) is equivalent to

$$
\begin{aligned}
& -\left(1+K_{+}\left(1-\theta_{0}\right)\right) \hat{c}_{k}-K_{+} d_{k}+K_{+} \tau_{N-k}^{+}(\mathbf{c})=0, k=1, \ldots, N-1, \\
& \left(1-K_{-}\left(1+\theta_{0}\right)\right) \hat{c}_{k}+K_{-} d_{k}+K_{-} \tau_{k}^{-}(\mathbf{c})=0, k=1, \ldots, N-1
\end{aligned}
$$

which can be uniquely solved by implicit function theorem since the matrix

$$
\left(\begin{array}{ccccc}
-\left(1+K_{+}\left(1-\theta_{0}\right)\right) & -K_{+} & 0 & \cdots & 0 \\
\left(1-K_{-}\left(1+\theta_{0}\right)\right) & K_{-} & 0 & \cdots & 0 \\
0 & & & \ddots & \\
0 & \ldots & 0 & -\left(1+K_{+}\left(1-\theta_{0}\right)\right) & -K_{+} \\
0 & \cdots & 0 & \left(1-K_{-}\left(1+\theta_{0}\right)\right) & K_{-}
\end{array}\right)
$$

is again nonsingular thanks to the nondegeneracy condition (1.4).

Then we define the new errors

$$
\bar{\lambda} u_{k}^{\prime}\left(t^{ \pm}\right)+K_{ \pm} u_{k}\left(t^{-}\right)-K_{ \pm} \log \left(\varepsilon^{2} \bar{\lambda}^{2}\right)=g_{k, \pm} \text { for } t=\mp \bar{\lambda}\left(1+ \pm \theta_{0}\right) \text {, respectively. }
$$

We get from (7.26) and (7.55)-(7.56) that

$$
\left|g_{k, \pm}\right| \leq C \varepsilon^{\mu}
$$

with some $\mu>0$.

The rest of the proof of Theorem 7.1 follows basically the steps of the proof of the special case in the previous section. Thus we have to consider the following linear system

$$
\vec{\phi}^{\prime \prime}=\left(\begin{array}{cccc}
2 e^{u_{1}} & -e^{u_{2}} & 0 \cdots & 0 \\
-e^{-u_{1}} & 2 e^{u_{2}} & -e^{u_{3}} \ldots & 0 \\
& & \ddots & \\
0 & \cdots & 2 e^{u_{N-2}} & -e^{u_{N-1}} \\
0 & \cdots & -e^{u_{N-2}} & 2 e^{u_{N-1}}
\end{array}\right) \vec{\phi}^{T}, \quad \vec{\phi}=\left(\phi_{1}, \ldots, \phi_{N-1}\right)
$$

We first observe that

$$
g_{j} \frac{\partial u_{k}}{\partial g_{j}}=\left\{\begin{aligned}
1, & j=k+1, \quad t \rightarrow \infty \\
-1, & j=k, \quad t \rightarrow \infty \\
1, & j=N+2-k, \quad t \rightarrow-\infty \\
-1, & j=N+1-k, \quad t \rightarrow-\infty \\
0, & \text { otherwise }
\end{aligned}\right.
$$

Hence by a transformation we can find a set of linearly independent solutions to (7.61)

$$
\psi_{1 k}(t)=\left\{\begin{aligned}
\vec{e}_{k}, & t \rightarrow \infty \\
-\vec{e}_{k}, & t \rightarrow-\infty
\end{aligned}\right.
$$


Similarly, considering derivatives of $u_{k}$ with respect to $w_{j}$ we can find solutions of $(7.61), \psi_{2 k}(t)$, $k=1, \ldots, N-1$ such that

$$
\psi_{2 k}(t)=\vec{e}_{k}|t|+O(1) .
$$

The functions $\left\{\psi_{1 k}(t), \psi_{2 k}(t)\right\}$ form a fundamental set for the system (7.61), whose behavior as $t \rightarrow \pm \infty$ is analogous to that of the functions $\psi_{1}(t), \psi_{2}(t)$, respectively.

Denoting by $\mathbf{u}_{0}(t)$ the solution to (7.43) where the parameters $\mathbf{w}_{0}$ and $\mathbf{g}_{0}$ satisfying (7.55)(7.56) and setting $u_{k}(t)=q_{k}(t)-q_{k+1}(t), \mathbf{u}=\left(u_{1}, \ldots, u_{N-1}\right)$, we look for the solution to (7.18)-(7.20) (expressed now in terms of $\mathbf{u}$ ) in the form

$$
\mathbf{u}(t)=\mathbf{u}_{0}(t)+\vec{\varphi}(t), \quad \vec{\varphi}=\left(\varphi_{1}, \ldots, \varphi_{N-1}\right)
$$

where the boundary conditions are given at $t=-\bar{\lambda}\left(1+\theta_{0}\right)$ and $t=\bar{\lambda}\left(1-\theta_{0}\right)$ with $\bar{\lambda}$ and $\theta_{0}$ defined in (7.26). By (7.60), $\mathbf{u}_{0}$ satisfies the required boundary condition up to order $O\left(\varepsilon^{\mu}\right)$, for some $\mu>0$. Following the scheme in the case $N=2$, using the fundamental set $\left\{\psi_{1 k}, \psi_{2 k}\right\}$, we can solve for the correction $\vec{\varphi}$. The rest of the proof is equally straightforward adaptation of the method of the previous section. We leave the details to the reader.

\subsection{Conclusion of the proof of Theorem 1.1}

To solve system (7.9)-(7.11) we will set up a fixed point argument scheme. To this end let $\tilde{\mathbf{f}} \in H^{1}(-1,1)$ be given and such that

$$
\|\tilde{\mathbf{f}}\|_{H^{1}(-1,1)} \leq|\log \varepsilon|^{2}
$$

and define for each $k=1, \ldots, N$

$$
h_{k}(\mathbf{f})=\mathcal{N}_{k 1}\left(\mathbf{f}, \mathbf{f}^{\prime}, \mathbf{f}^{\prime \prime}\right)+\mathcal{N}_{k 2}\left(\tilde{\mathbf{f}}, \tilde{\mathbf{f}}^{\prime}\right) .
$$

With this $\mathbf{h}=\left(h_{1}, \ldots, h_{N}\right)$ we can use Theorem 7.1 to solve, now nonlinear, problem

$$
\begin{aligned}
\alpha_{0} \sqrt{2} \varepsilon^{2} f_{k}^{\prime \prime}-e^{-\sqrt{2}\left(f_{k}-f_{k-1}\right)}+e^{-\sqrt{2}\left(f_{k+1}-f_{k}\right)}= & h_{k}(\mathbf{f}), \\
& -1<\theta<1, \\
f_{k}^{\prime}(-1)+K_{-} f_{k}(-1)= & 0, \\
f_{k}^{\prime}(1)+K_{+} f_{k}(1)= & 0 .
\end{aligned}
$$

Here it is important that $\mathcal{N}_{k 1}$ is a contraction in $H^{2}(-1,1)$ and therefore, making use of the theory developed above for the linearized Toda system and employing Banach Contraction Mapping theorem we find $\mathbf{f}$ given $\tilde{\mathbf{f}}$. This way we define a mapping $\mathcal{T}(\tilde{\mathbf{f}})=\mathbf{f}$ and the solution of our problem is simply a fixed point of $\mathcal{T}$. Continuity of $\mathcal{N}_{k i}, i=1,2$, with respect to its parameters and a standard regularity argument allows us to conclude that $\mathcal{T}$ is compact as a mapping from $H^{1}(-1,1)$ into itself. Then Schauder Theorem applies to yield the existence of a fixed point of $\mathcal{T}$ as required. Finally, we should remark that uniqueness of the solutions found for system (7.62)-(7.64) for $h_{k}=0$ implies the symmetry fact $f_{k}=-f_{N-k}$. This symmetry is thus approximately inherited for the inhomogeneous perturbed problem. The construction itself provides the validity of the asymptotic expressions (1.18). This ends the proof of Theorem 1.1.

Acknowledgments: The first author has been partly supported by research grants Fondecyt 1030840 and FONDAP, Chile. The second author has been supported by Fondecyt grant 1050311 and Nucleus Millennium grant P04-069-F. The research of the third author is partially supported by an Earmarked Grant from RGC of Hong Kong. 


\section{References}

[1] N. Alikakos, X. Chen And G. Fusco, Motion of a droplet by surface tension along the boundary, Cal. Var. PDE, Vol. 11, no 3 (2000), 233-306.

[2] S. Allen And J. W. CAhn, A microscopic theory for antiphase boundary motion and its application to antiphase domain coarsening, Acta. Metall. 27 (1979), 1084-1095.

[3] L. Bronsard, R. V. Kohn, Motion by the mean curvature as the singular limit of Ginzburg-Landau dynamics, J. Differential Equations, 90 (1992), pp. 211-237.

[4] L. Bronsard And B. Stoth, On the existence of high multiplicity interfaces, Math. Res. Lett. 3 (1996) 117-151.

[5] M. BARdi, B. Perthame, Exponential decay to stable states in phase transitions via a double log-transformation. Comm. Partial Differential Equations 15 (1990), no. 12, 1649-1669.

[6] L. CAfFarelli, A. Cordoba Uniform convergence of a singular perturbation problem. Comm. Pure Appl. Math. 48 (1995), no. 1, 1-12.

[7] R. Casten, C.J. Holland, Instability results for reaction diffusion equations with Neumann boundary conditions. J. Differential Equations 27 (1978), no. 2, 266-273.

[8] X. CHEn, Generation and propagation of interfaces in reaction-diffusion equations, J. Diff. Eqns. 96 (1992), 116-141.

[9] P. De Mottoni, M. Schatzman, Geometrical evolution of developed interfaces, Trans. Amer. Math. Soc. 347, No. 5 (1995) 1533-1589.

[10] M. Del Pino, P. Felmer, K. Tanaka, KazunagaAn elementary construction of complex patterns in nonlinear Schrodinger equations. Nonlinearity 15 (2002), no. 5, 1653-1671.

[11] M. Del Pino, J. Dolbeault, M. Musso, "Bubble-tower" radial solutions in the slightly supercritical Brezis-Nirenberg problem. J. Differential Equations 193 (2003), no. 2, 280-306.

[12] M. Del Pino, M. Kowalczyk, J. Wei, Concentration on curves for nonlinear Schrödinger equations, Comm. Pure Appl. Math. 60 (2007) no. 1, 113-146.

[13] M. del Pino, M. KowalczyK, X. Chen, The Gierer $\&$ Meinhardt system: the breaking of homoclinics and multi-bump ground states. Commun. Contemp. Math. 3 (2001), no. 3, 419-439.

[14] S.-I. Ei And E. YanagidA, Slow dynamics of interfaces in the Allen-Cahn equation on a strip-like domain, SIAM J. Math. Anal. Vol. 29, No. 3 (1998), 555-595.

[15] L. C. Evans, H. M. Soner And P. E. Souganidis, Phase transition and generalized motion by mean curvature, Comm. Pure Appl. Math. 45 (1992), 1097-1123.

[16] C.E. Garza-Hume, P. Padilla, Closed geodesics on oval surfaces and pattern formation. Comm. Anal. Geom. 11 (2003), no. 2, 223-233.

[17] J.E. Hutchinson, Y. Tonegawa, Convergence of phase interfaces in the van der WaalsCahn-Hilliard theory. Calc. Var. Partial Differential Equations 10 (2000), no. 1, 49-84

[18] T. Ilmanen, Convergence of the Allen-Cahn equation to the Brakke's motion by mean curvature, J. Diff. Geom 38 (1993), 417-461.

[19] J. Jost, G. WAng, Classification of solutions of a Toda system in $\mathbb{R}^{2}$. Int. Math. Res. Not. 2002, no. 6, 277-290.

[20] X. KANG, J. WEI, On interacting bumps of semi-classical states of nonlinear Schrodinger equations. Adv. Differential Equations 5 (2000), no. 7-9, 899-928 
[21] M. Katsoulakis, G.T Kossioris And F. Reitich, Generalized motion by mean curvature with Neumann conditions and the Allen-Cahn model for phase transitions, J. Geom. Anal. Vol 5, No 2 (1995), 255-279.

[22] R. V. Kohn And P. Sternberg, Local minimizers and singular perturbations, Proc. Royal Soc. Edinburgh 111 A (1989), 69-84.

[23] B. Konstant, The solution to a generalized Toda lattice and Representation Theory, Adv. Math. 34 (1979), 195-338.

[24] M. KowalczyK, On the existence and Morse index of solutions to the Allen-Cahn equation in two dimensions, Ann. Mat. Pura Appl. 1844 (2005), no. 1, 17-52.

[25] A. Malchiodi, M. Montenegro, Boundary concentration phenomena for a singularly perturbed elliptic problem. Comm. Pure Appl. Math. 55 (2002), no. 12, 1507-1568.

[26] A. Malchiodi, M. Montenegro, Multidimensional boundary layers for a singularly perturbed Neumann problem. Duke Math. J. 124 (2004), no. 1, 105-143.

[27] A. Malchiodi, W.-M. Ni, J. Wei, Multiple clustered layer solutions for semilinear Neumann problems on a ball. Ann. Inst. H. Poincare Anal. Non Lineaire 22 (2005), no. $2,143-163$.

[28] A. Malchiodi, W.-M. Ni, J. WeI, Boundary clustered interfaces for the Allen-Cahn equation. Preprint 2005.

[29] L. ModicA, The gradient theory of phase transitions and the minimal interface criterion. Arch. Rational Mech. Anal. 98 (1987), no. 2, 123-142.

[30] H. Matano, Asymptotic behavior and stability of solutions of semilinear diffusion equations. Publ. Res. Inst. Math. Sci. 15 (1979), no. 2, 401-454.

[31] J. Moser Finitely many mass points on the line under the influence of an exponential potential-an integrable system. Dynamical systems, theory and applications (Rencontres, BattelleRes. Inst., Seattle, Wash., 1974), pp. 467-497. Lecture Notes in Phys., Vol. 38, Springer, Berlin, 1975.

[32] K. NAKASHIMA, Multi-layered stationary solutions for a spatially inhomogeneous AllenCahn equation. J. Differential Equations 191 (2003), no. 1, 234-276.

[33] K. NAKASHIMA AND K. TANAKA, Clustering layers and boundary layers in spatially inhomogeneous phase transition problems, Ann. Inst. H. Poincare Anal. Non Lineaire 20 (2003), no. 1, 107-143.

[34] F. PACARD AND M. RitorÉ, From the constant mean curvature hypersurfaces to the gradient theory of phase transitions, J. Differential Geom. 64 (2003), no. 3, 359-423.

[35] P. Padilla And Y. Tonegawa, On the convergence of stable phase transitions, Comm. Pure Appl. Math. 51 (6) (1998), 551-579.

[36] J. Rubinstein, P. Sternberg And J.B. Keller, Fast reaction, slow diffusion and curve shortening, SIAM J. Appl. Math, Vol 49, No. 1 (1989), 116-133.

[37] H. M. Soner, Ginzburg-Landau equation and motion by mean curvature, Part I: Convergence, Part II: Development of the initial interface, Jour. Geom. Anal. 7, (3) (1997), Part I 437-455, Part II 477-491.

[38] P. Sternberg, The effect of a singular perturbation on nonconvex variational problems. Arch. Rational Mech. Anal. 101 (1988), no. 3, 209-260.

[39] P. Sternberg, K. Zumbrun, Connectivity of phase boundaries in strictly convex domains. Arch. Rational Mech. Anal. 141 (1998), no. 4, 375-400.

[40] Y. Tonegawa Phase field model with a variable chemical potential Proc. Roy. Soc. Edinburgh Sect. A 132 (2002), no. 4, 993-1019. 